\section{JUI 29997 \\ OSTI}

ANL-HEP-PR-95-58

ZEUS-Note 95-064

May 25, 1995

\title{
Studies of Hadron-Electron Separators for the ZEUS Barrel Calorimeter
}

\author{
I.A.MBats, D.Bortz ${ }^{1}$, A.CONNOLlY ${ }^{2}$, A.DerlickI ${ }^{3}$, M.Derrick, W.KaHLe, \\ S.MAGILl, D.MIKUNAS ${ }^{4}$, B.Musgrave, J.Schlereth, R.STANEK, AND \\ J.THRON \\ Argonne National Laboratory \\ Argonne, IL 60439, U.S.A.
}

\begin{abstract}
Two possible upgrades, a shower maximum detector and a presampler, designed to improve the low energy electron/hadron separation capabilities of the ZEUS barrel calorimeter are described and test-beam results are reported. The presampler can also be used to correct for energy loss of particles traversing the dead material in front of the calorimeter.
\end{abstract}

\section{Introduction and Motivation for the BHES}

ZEUS is a detector used to study high energy e-p scattering at the HERA collider located at the DESY Laboratory. The High Resolution Calorimeter of ZEUS, which uses alternating layers of depleted uranium and scintillator, has outstanding energy resolution for jets and gives good measurements of the recoil electron in deep inelasic scatiering when the latter is isolated. However, the limited number of readout channels that is characteristic of scintillator based calorimeters makes the detection of electrons and photons within jets difficult.

\footnotetext{
${ }^{1}$ Summer 1994 Student Research Participation Program.

${ }^{2}$ Argonne Research Aide Appointee.

${ }^{3}$ now at University of Warsaw.

*Also at Illizois Institute of Technology.
}

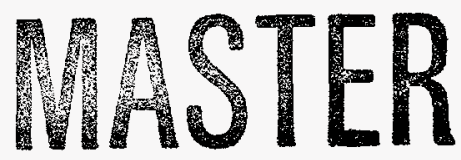

The womitted menuscript hes been outhored or contrector of the U.S. Government under contract No. W-3i-100-ENG-38. Accordingly, th U.S Governmome retsine a

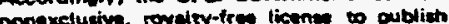
mor rem of this or reprodices the publinhed torm of this contribution, or allom otwere to do so, for 


\section{DISCLAMMIER}

Portions of this document may be illegible in electronic image products. Images are produced from the best available original document. 
The calorimeter is divided into three sections called the forward (FCAL) [1] [2], barrel (BCAL) [3], and rear (RCAL) [2]. Each section is divided longitudinally with segments read out separately; an electromagnetic (EMC) and two hadronic segments (HAC1) and (HAC2), or, in the case of the RCAL, an electromagnetic and one hadronic segment.

To improve the effective transverse segmentation, the RCAL has been equipped with a set of $3 \mathrm{~cm}$ square silicon pads placed near the maximum of the electromagnetic showers in the EMC section of the calorimeter. This device is called the Hadron Electron Separator (HES) [4]. An essentially identical system is planned for the FCAL. It is natural, therefore, to consider a similar augmentation of the BCAL.

The HES is used to provide a finer segmentation, and so a better precision in the location of electromagnetic showers than is allowed by the $10 \mathrm{~cm} \times 20 \mathrm{~cm}$ $(5 \mathrm{~cm} \times 20 \mathrm{~cm})$ size of the RCAL (FCAL) scintillator towers. This finer segmentation is particularly important when more than one particle is incident on the same tower, as is often the case when the electrons result from the semileptonic decay of charm and bottom quarks. Identification and measurement of single photons and their discrimination from $\pi^{0}$ 's is also important in measuring direct photon production processes. Such problems are expected to be more severe in the FCAL and RCAL than in the BCAL, the FCAL because of the boost effects and the RCAL because of the EMC tower size.

A HES consisting of silicon pads does a good job, but is expensive on the scale of $\sim 10 \mathrm{~m}^{2}$ which characterises the FCAL or RCAL. Since the surface area of the $B C A L$ is equal to the sum of those of the RCAL and FCAL; we have pursued the simplez anc cheaper option for a barzel HES (BHES) of a proportional wire chamber system. The design uses a set of wires along $z$, the e-p beam direction, and a matrix of pads in the orthogonal or $\phi$ direction. Such a system is used with the CDF detector [5]. An alternative technology that uses bundles of scintillating fibers to measure the $\phi$ coordinate has been tested by a group from the Ohio State University [6].

To correct for energy loss of particles in the dead material between the e-p 
interaction point and the calorimeter, a layer of scintillator that is separately read out can be used as a presampler [7]. Such a presampler can also be used to help separate electrons from pions.

This paper presents the results of tests of both a presampler and a wirechamber shower-maximum detector used with the prototype BCAL module, exposed to electron and pion beams of momentum $1-6 \mathrm{GeV} / \mathrm{c}$ at the Brookhaven Alternating Gradient Synchrotron.

\section{The HES Design}

\subsection{Mechanical}

The proposed BHES utilizes wire chamber technology with anode wire and cathode pad readout. The basic structure consists of eight individual PWC cells created by wires running between ribs of an aluminum extrusion, as shown in Figure 1 . An extrusion contains two side walls, a bottom wall and seven isolating ribs. Each extrusion is $2900 \mathrm{~mm}$ long, $57 \mathrm{~mm}$ wide and $10 \mathrm{~mm}$ high. Plastic manifolds at each end direct the gas flow in series through each cell. The top, open, surface is closed with five segmented printed circuit boards that provide readout of the cathode pad signals. There are four such structures (skis) per BCAL module. Within each module, after three uranium plates $\left(3 X_{0}\right)$, a space was reserved during the construction of the modules for the installation of the four skis.

The EMC cells in the BCAL have dimensions of $250 \mathrm{~mm}$ in the $\phi$ and $50 \mathrm{~mm}$ in the $z$ direction. The eight anode wires of each ski, which are parallel to the ZETS $z$-axis, measure the azimuthal angle $(\phi)$, while the 160 cathode pads along the $z$-axis span the eight wires and measure the polar angle $(\theta)$. The spacing between wires is $6.8 \mathrm{~mm}$ and the spacing between pad centers is $17 \mathrm{~mm}$, so that three pads cover each EMC cell in the $\mathrm{z}$ direction. Each cell has a cross sectional area of $5.8 \mathrm{~mm}$ wide by $5.5 \mathrm{~mm}$ high. The anode wires have a diameter of $50 \mu \mathrm{m}$ and are made of gold 


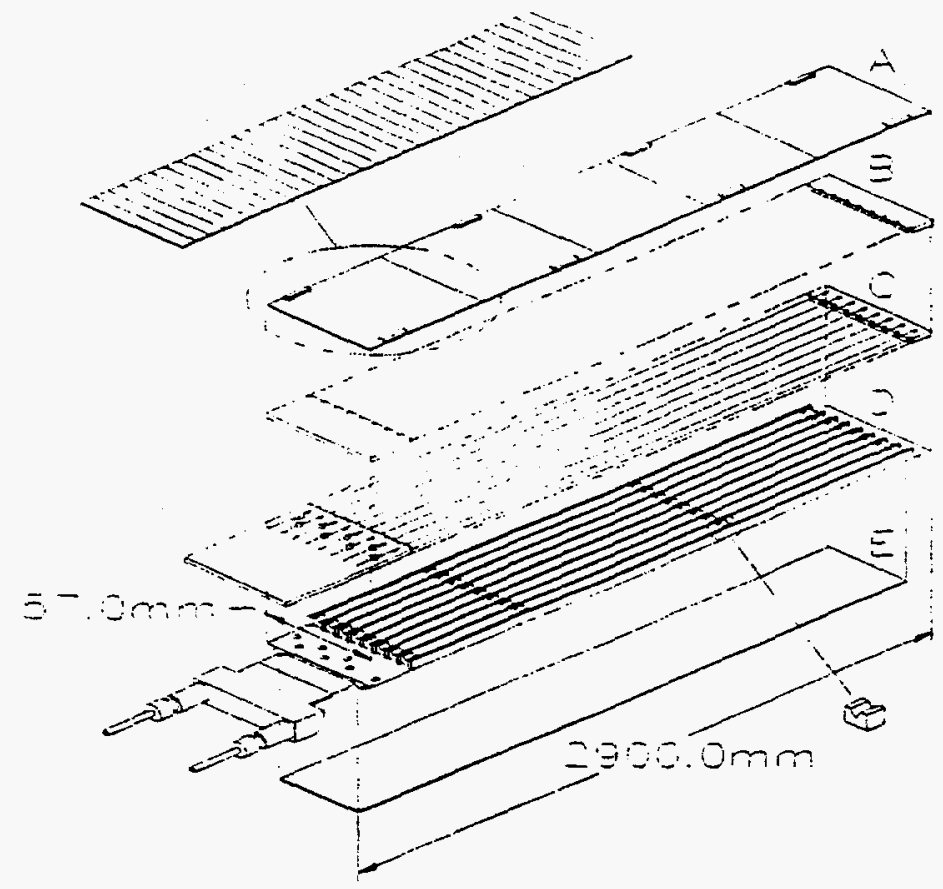

Figure 1: Exploded view of a BHES ski. The five printed circuit boards with one board enlarged to make the 16 pads per board visible are shown in layer $A$. The endcaps that seal the extrusions are shown in layer B. The eight wires per ski are shown in layer C. The extrusion that forms the eight individual chambers, the gas manifold and an enlarged support are shown in layer $\mathrm{D}$. The box beam is shown in layer $E$. Note that the long dimension is not drawn to scale.

plated tungsten. They are stretched to a tension of $250 \mathrm{~g}$ and are supported along their length at two asymmetric points, $560 \mathrm{~mm}$ from one end and $1110 \mathrm{~mm}$ from the other end. The supports, delrin inserts, were snug fit between the ribs and the wires were epoxied to them. Individual wires are connected to high voltage through $20 \mathrm{M} \Omega$ resistors and readout through $220 \mathrm{pF}$ capacitors using a printed circuit board located at one end of each ski. A gas mixture of $95 \%$ argon and $5 \% \mathrm{CO}_{2}$ was used during the beam tests. Bench tests showed negligible gas leaks.

Five 3-layer PC cathode boards, $55 \mathrm{~mm}$ wide and $557 \mathrm{~mm}$ long, are glued to the open side of the extrusions and connected with soldered joints to provide a 


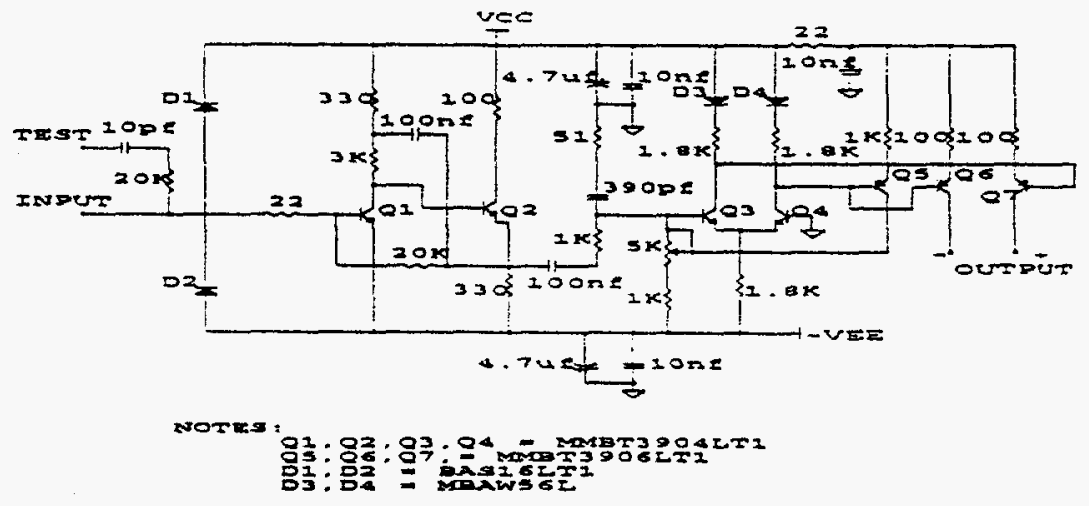

Figure 2: Schematic of the wire amplifier/driver circuit.

gas seal between the boards. The pads are electrically insulated from the ribs of the extrusion with a solder mask on the PC boards.

The BHES contains a dead area of about $20 \%$ and a fully instrumented ski corresponds to approximately $10 \%$ of a radiation length. The dead area produced by the ribs is only a problem for single minimum ionizing particles, such as muons or late showering hadrons. Electromagnetic and hadronic showers in the BCAL EMC (BEMC) section span several wires.

\subsection{Electronics}

Short microstrip cables bring the anode wire signals to an amplifier board located at the rear end of the BCAL. Figure 2 shows the design of the wire amplifier/driver circuit.

The cathode boards have pads etched on the inside surface, with vias to the outside surface where surface mount preamplifiers shape the signals and drive mi- 


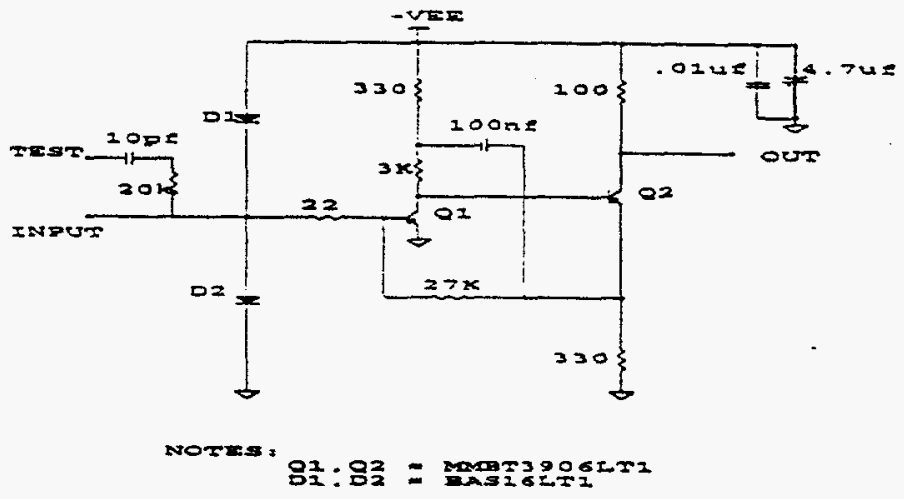

Figure 3: Schematic of the pad preamplifier.

crostrip cables running along the length of the ski. These cables bring the signals to the end of each ski, at the rear of the BCAL. Figure 3 shows the design of the pad preamp. The microstrip lines are fed into five amplifier/summer/driver cards located at the rear end of each BEMC module, where the signals are amplified further.

Since there is a low multiplicity of particles coming from the HERA e-p interactions in any BCAL module, pads from the four skis in each module at the same $z$ are further ganged together to form 160 strips per module, as shown in Figure 4. Thus the cathode segmentation in $\phi$ is 11.25 degrees, the same as a BCAL module. Finer segmentation in $\phi$ is provided by the anode wires. The summing of the pad signals is done on the five cards located at the rear of the module. The driver cards send the 160 pad signals and the 32 wire signals down twelve 34-conductor twisted pair cables to the readout electronics. Figure 4 shows the design of the pad summer and driver boards.

Both wire and strip preamps are based on a simple and inexpensive current amplifier with positive feedback to increase the dynamic range and linearity. The 


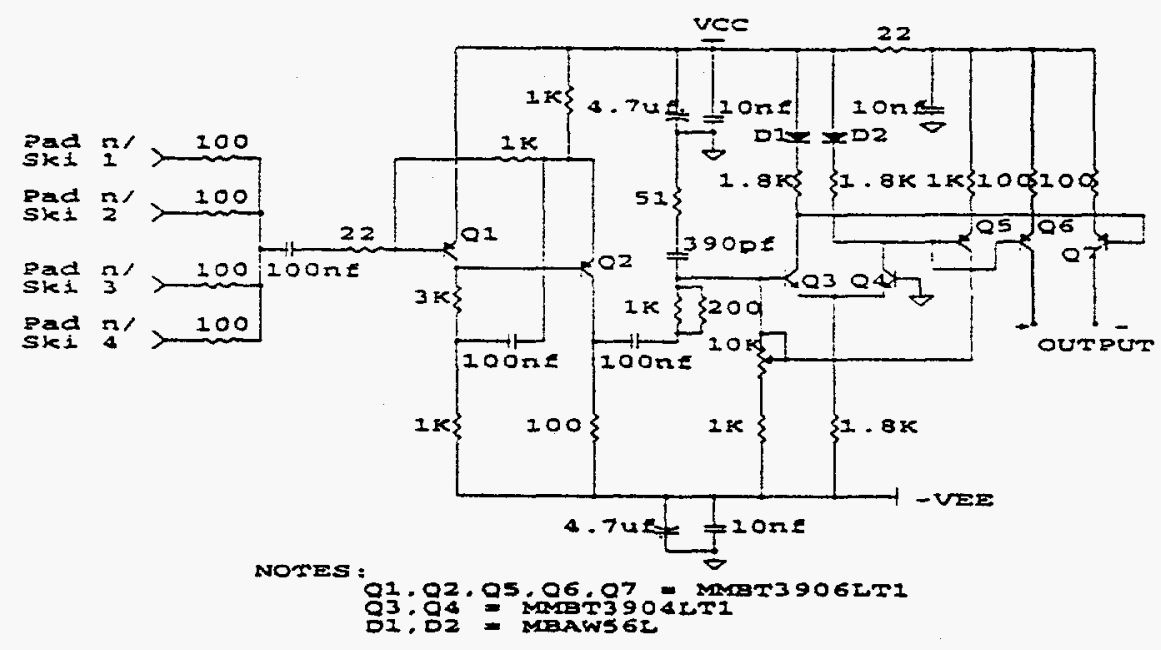

Figure 4: Schematic of the pad summer/driver circuit.

current gain of the amplifiers is 450 and the dispersion is $<5 \%$ for 600 tested channels. The dynamic range is 200 , and the non-linearity is $<1 \%$. Figure 5 shows the mean charge on a wire for electron showers in the energy range of $1-6 \mathrm{GeV}$. The vertical bars shown on the figure correspond to the RMS of the charge distributions. The dynamic range of the electronics is set to accommodate the maximum fluctuations.

Power dissipated in the cathode preamplifiers along the skis is less than 10 watts per BCAL module, and the power dissipated in the driver cards at the rear of a BCAL module is 20 watts. In addition to the twisted-pair cables and the gas lines to the skis, each module receives one high voltage cable, one 10-conductor charge injection cable and four wires carrying \pm 5 volts. 


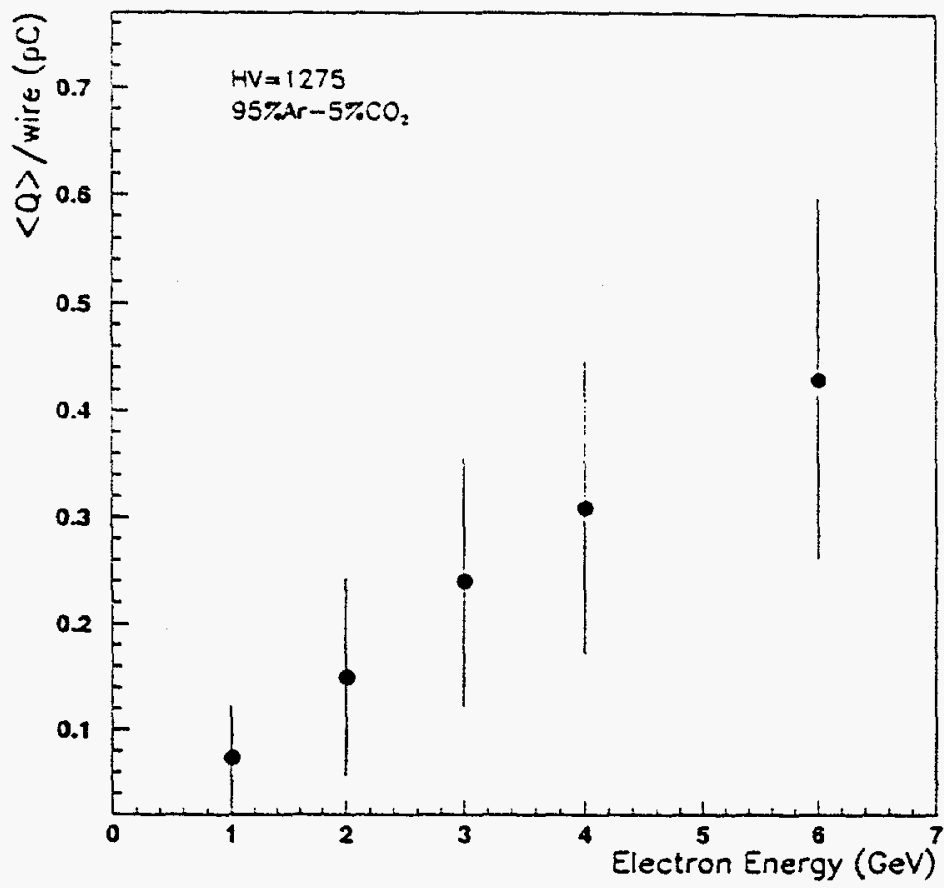

Figure 5: The mean charge per BHES wire for electron showers. The operating voltage is 1275 Volts and the gas mixture is $95 \% \mathrm{Ar}-5 \% \mathrm{CO}_{2}$. The vertical bars on the data points indicate the RMS widths of the distributions.

\section{Testbeam Setup and Readout Electronics.}

The BNL testbeam provided 1 - $6 \mathrm{GeV} / \mathrm{c}$ electrons, pions, protons and muons produced from the AGS proton beam. A schematic of the detector setup in the test beam is shown in Figure 6. The beam was defined through the coincidence of two scintillation counters S2 and S3. To locate the beam with greater accuracy, the coincidence between vertical and horizontal crossed finger scintillation counters, SA and SB, each $1 \mathrm{~cm}$ wide, was used to define a beam area. To reject events with halo particles, the $1 \mathrm{~cm}^{2}$ beam area was surrounded by four large scintillation counters included in the trigger as vetos (VETO). Two threshold Cerenkov counters (C1,C2) were provided and one ( $\mathrm{C} 2$ ) was used to discriminate between electrons and heavier particles. The 
trigger logic used to select electrons was: $S 2 * C 2 * S 3 * \overline{V E T O} * S A * S B$. To select heavier particles $C 2$ was replaced with $\overline{C 2}$. Muons were identified offline by their characteristic minimum ionizing signal in the HAC sections of the BCAL.
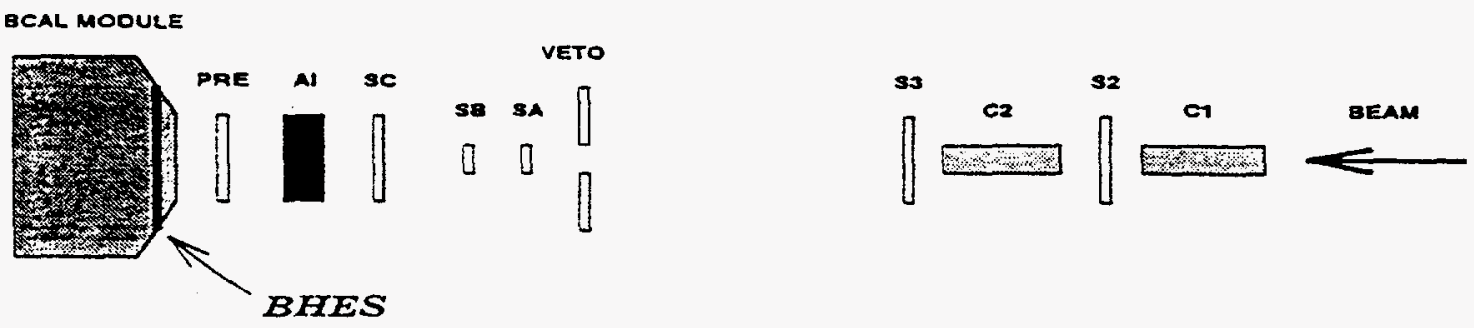

Figure 6: Testbeam Schematic; Top View.

In the right-handed cartesian coordinate system at the test beam, the beam defined the $x$-axis and the $z$-axis was horizontal. Therefore, the testbeam $y$ coordinate corresponds to the ZEUS $\phi$ angle and the testbeam and ZEUS $\mathrm{z}$ coordinates are the same. The prototype BCAL module was placed horizontally in the beam on a stand [8] which allowed the module to be moved in the vertical or horizontal directions with a location accuracy of $0.5 \mathrm{~mm}$. To mimic the dead material between the interaction point and the calorimeter at ZEUS, a $1 \mathrm{X}_{0}$ aluminum block was placed at the appropriate distance in front of the BCAL module. A scintillation counter, SC, was placed directly upstream of this absorber to tag non-showering incident particles. Another scintillation counter, PRE, was placed directly downstream of the aluminum to measure the total charge exiting the dead material. The light yield of this counter was 73 photoelectrons per minimum ionizing particle (mip).

The differential signals from the skis were received by active line receivers which then drove $50 \Omega$ into Lecroy $2249 \mathrm{~W}$ ADC's. The trigger electronics generated a 600 nsec gate and the 32 wire and 16 pad signals were digitized and read out with a GPIB camac interface to an IBM-PC. 


\section{Calibration of the BCAL Prototype}

The uranium/scintillator calorimeter is inherently self monitoring through the uranium radioactivity and the resulting PMT current. These currents were used to set the PMT high voltage uniformly. Two methods were used to give an absolute calibration of the energy scale of the BCAL module.

1) From the measured response of the calorimeter to muons present in the beam.

2) By using the known beam momentum for electrons incident on the module. This method can only be used for the EMC sections. The pion data was not used since the transverse size of the module is too small to completely contain the pion induced hadronic shower.

The BCAL modules have been extensively calibrated using muons, both at a high energy test beam at Fermilab [8] and on a cosmic ray test stand at Argonne [9]. Low energy muons were also used at CERN [2] to calibrate the FCAL and RCAL modules of the ZEUS calorimeter. These have the same uranium-scintillator sandwich construction as does the BCAL.

In the BNL test beam, the muons and pions were selected by a trigger which used $\mathrm{C} 2$ set at muon threshold, thus vetoing electrons. In the triggered pion data, the fraction of muons present was $\sim 3 \%$. Muons were distinguished from pions using the $\mathrm{HAC2}$ section of the calorimeter since, for most pions, interactions started in the uranium absorber well before the HAC2 section and were fully contained longitudinally in the EMC and $\mathrm{HACl}$ sections. Muons, which fully traverse the BCAL provide a signal corresponding to an energy deposition of about $700 \mathrm{MeV}$ in $\mathrm{HAC2}$. This appears as an excess of events on the falling pion signal distribution. The distribution was fit with a gaussian plus polynomial, which gave the position of the muon peak and so the mean charge deposited by the muons. The events that were contained in the muon peak in HAC2 were then examined in the HAC1 and the EMC sections to determine the muon charge deposited in each of these sections. 
To calibrate the EMC and $\mathrm{HACl}$ cells which the muon beam did not pass through directly, the PMT current produced by the radioactivity of the depleted uranium, the DU current, was used. The calibration charge, $Q_{\text {cal }}$, for a cell not illuminated by muons was related to its corresponding cell muon charge, $Q_{\text {mwon }}$, through $R$, the ratio of the $\mathrm{DU}$ current in the uncalibrated cell to the $\mathrm{DU}$ current in the muon calibrated cell, i.e., $Q_{\text {cal }}=R \times Q_{\text {munn }}$. Using this relationship, the cells not directly illuminated by muons were cross calibrated to the muon signal.

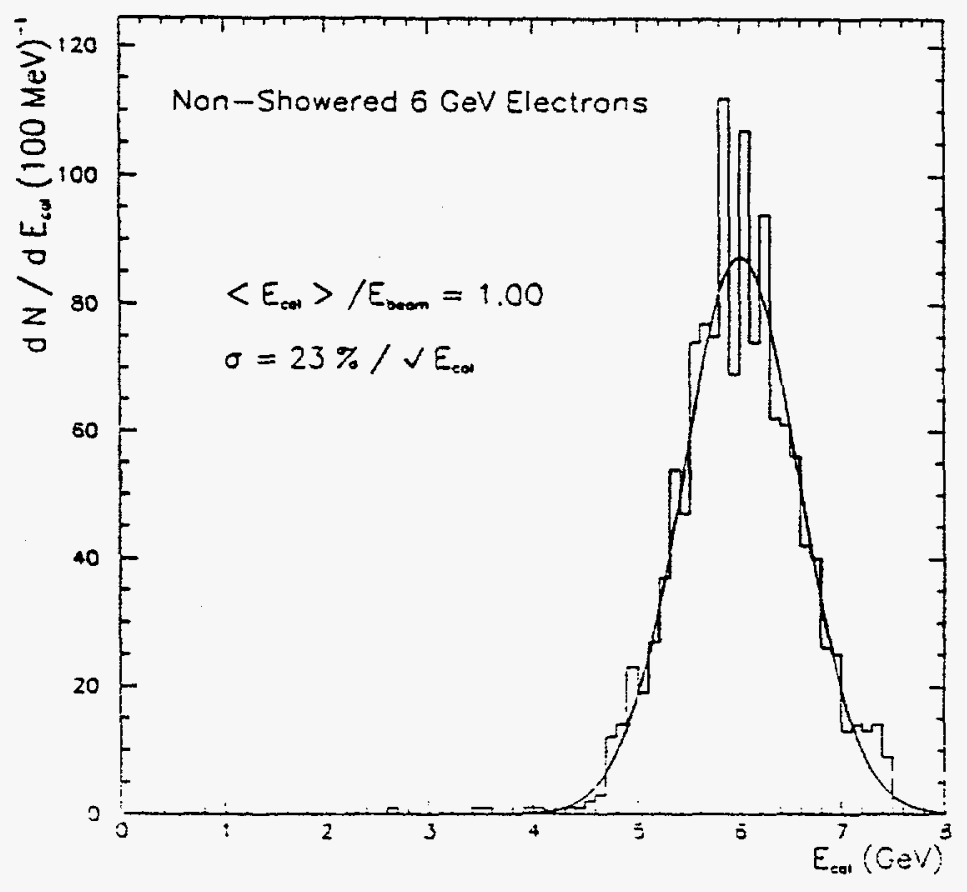

Figure 7: BCAL measured energy for $6 \mathrm{GeV} / \mathrm{c}$ electrons after calibrating using muons. The error on the mean measured energy, $E_{c a l}$, is $\pm 2 \%$.

To determine the calorimeter energy scale, the relationship between energy deposited in the calorimeter and the observed charge was obtained using the known muon $\mathrm{dE} / \mathrm{dx}$ relationship and the number and properties of the layers of absorber and scintillator. A typical calibration constant for a $\mathrm{BAC}$ cell was $\sim 10.5 \mathrm{pc} / \mathrm{GeV}$ and for EMCs, $\sim 8.5 \mathrm{pc} / \mathrm{GeV}$. Figure 7 shows the energy measured in the BCAL 
module for non-showered electrons ( $<2$ mips seen in PRE) of momentum $6 \mathrm{GeV} / \mathrm{c}$. The mean of the fit to the measured value agrees to within $2 \%$ with the known test beam momentum. The width of the measured distribution corresponds to a resolution of $23 \% / \sqrt{E}$, consistent with the known relatively low light output of the prototype module.

\section{BHES Calibration}

The HES was calibrated using both a ${ }^{109} \mathrm{Cd}$ source and a precision charge injector. As seen in Figures 2 and 3, precision $10 \mathrm{pf}$ capacitors are used to store a fixed charge for any given channel. Relative gains between channels, for both pads and wires, were measured with this technique.

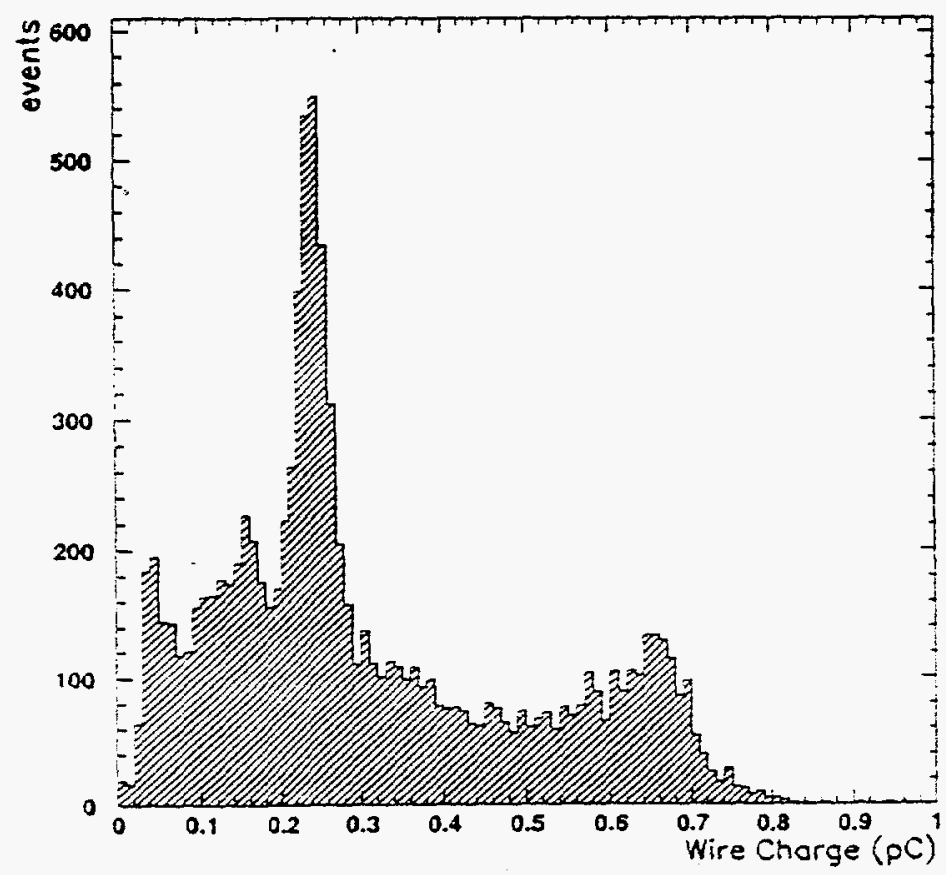

Figure 8: Observed wire pulse height spectrum for a ${ }^{109} \mathrm{Cd}$ source. 
The absolute gains of the chamber wires and the chamber pads were calibrated using a ${ }^{109} \mathrm{Cd}$ source. The spectrum of ${ }^{109} \mathrm{Cd}$ decay products and their interaction in the material of the PWC is rather complicated. The dominant feature in the HES response results from the $23 \mathrm{keV} \gamma$-ray, emitted by the ${ }^{109} \mathrm{Cd}$, interacting in the copper pads to produce an $8.02 \mathrm{keV} \mathrm{x}$-ray which is then absorbed in the gas. This produces the main peak seen in the wire pulse height distribution of Figure 8.

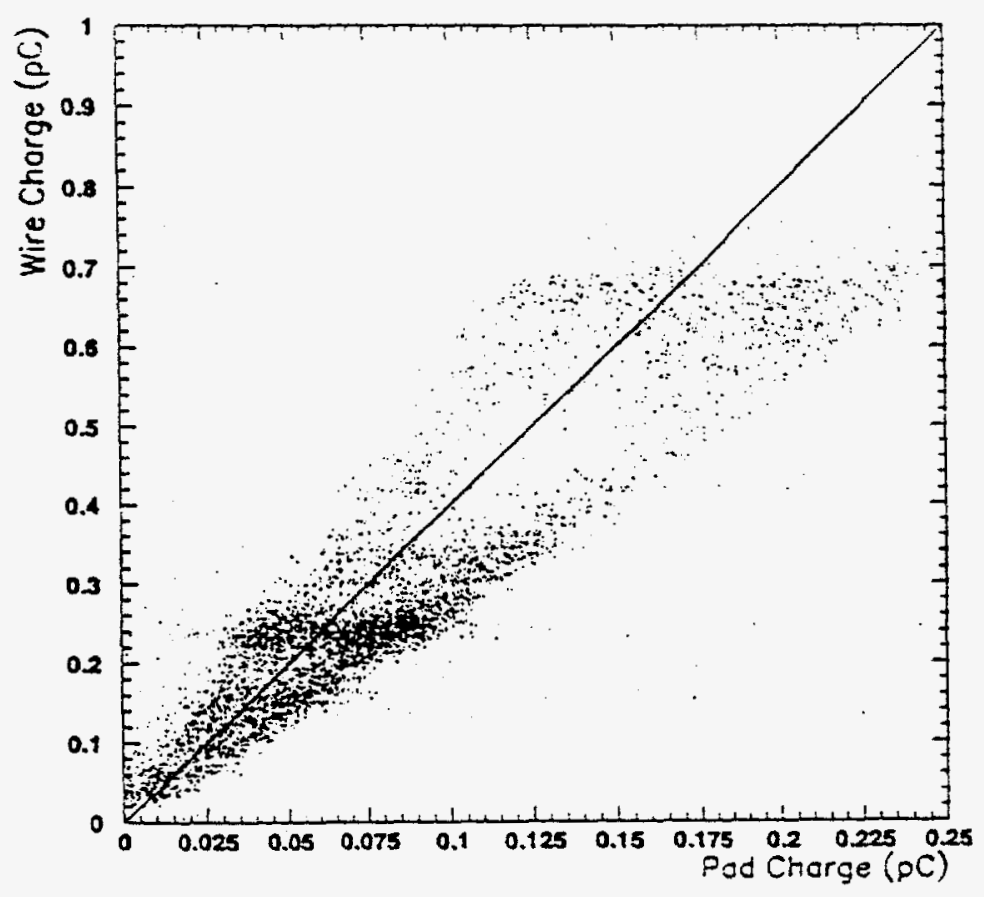

Figure 9: Wire vs pad pulse height for ${ }^{109} \mathrm{Cd}$ signals. The two distinct diagonal bands correspond to photons absorbed below and above the anode wire with the larger pad charges occuring near the pad. The diagonal line with a slope of four lies in the center of these two bands.

Furthermore, from the geometry, we expect that the pad charge should be $25 \%$ of the wire charge. Figure 9 shows the correlation between the pad charge and the wire charge. The two distinct bands are a consequence of the $8.02 \mathrm{keV}$ photons interacting above and below the wire, with a corresponding effect on the pad signal. The average ratio of wire to pad charge is four, as expected on geometrical grounds. 
The gain of the wire chamber is then given, assuming that the $8.02 \mathrm{keV}$ photon generates 290 primary ion pairs [10]. From our source measurements with $80 \% \mathrm{Ar}$ $20 \% \mathrm{CO}_{2}$ at a high voltage of $1550 \mathrm{~V}$, we find the wire gain to be $5.2 \cdot 10^{3}$. A mip is expected to create 50 ion pairs. These operating parameters result in a mean wire response of about $0.04 \mathrm{pC} / \mathrm{mip}$. The beam tests were performed with $95 \% \mathrm{Ar}-5 \% \mathrm{CO}_{2}$ and at an operating voltage of 1275 - 1365 volts, making a direct comparison of source and testbeam data difficult. Source scans were also used to map the wire gains and the pad-wire ratios were in turn used to map the pad gains.

\section{Presampler Results}

\subsection{Calorimeter Energy Measurement Corrections with a Presampler}

Due to the presence of material in the beamline, including the air through which the beam traveled as well as the aluminum blocks placed in the beamline to simulate the ZEUS solenoidal magnet, some beam particles lost energy by showering before entering the calorimeter cells. These pre-showered particles were detected in the scintillator paddle PRE placed immediately upstream of the calorimeter face. Figure 10 shows the ADC signal from this counter for $3 \mathrm{GeV}$ electrons and pions.

Clearly prominent in the electron spectrum is the peak representing nonshowered, single mip electrons, but there is also a large tail of multi-mip signals from showered electrons. The energy loss reflected in the calorimeter measurement due to pre-showering can be corrected in a straightforward way by comparing the measured calorimeter energy to the presampler signal. Figure 11 shows the measured calorimeter energies versus the presampler signals for electrons passing through the $1 X_{0}$ aluminum block. An offset in the first bin is observed for all but the $6 \mathrm{GeV}$ electrons, which is presumably due to loss of low energy particles in the aluminum. Note also that the slopes of the straight line fits to the data are approximately equal. 


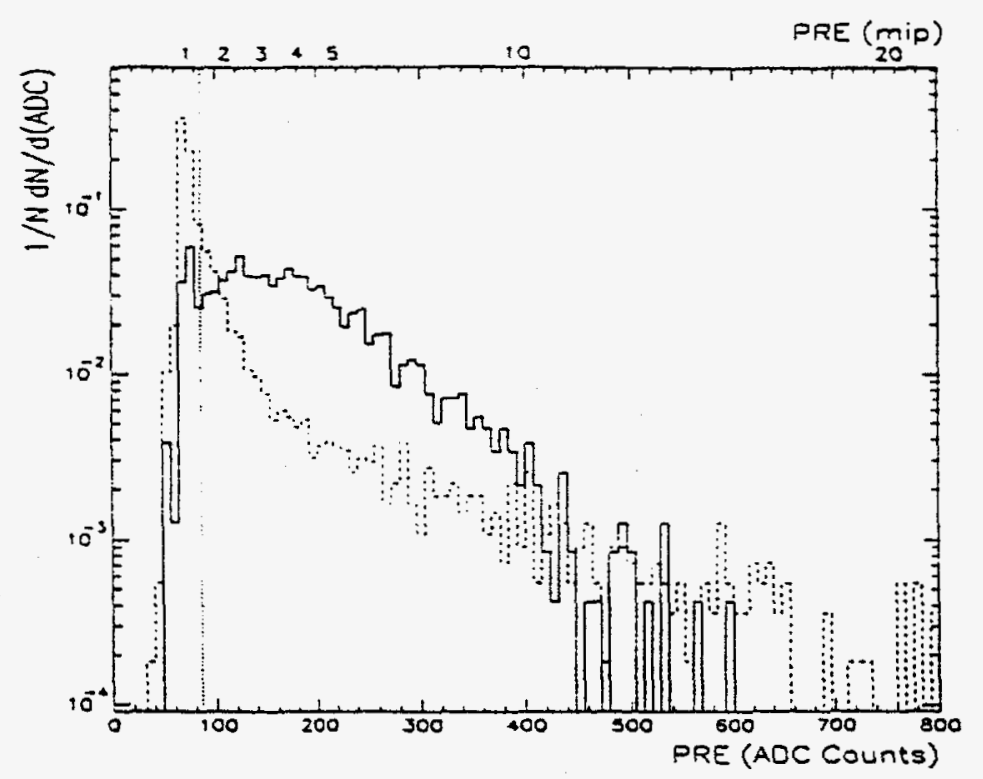

Figure 10: Observed ADC signal in PRE for $3 \mathrm{GeV}$ electrons (solid) and pions (dashed). The top axis label indicates bins calibrated in mips with the dotted line separating the 1 mip signal from the multi-mip signal.

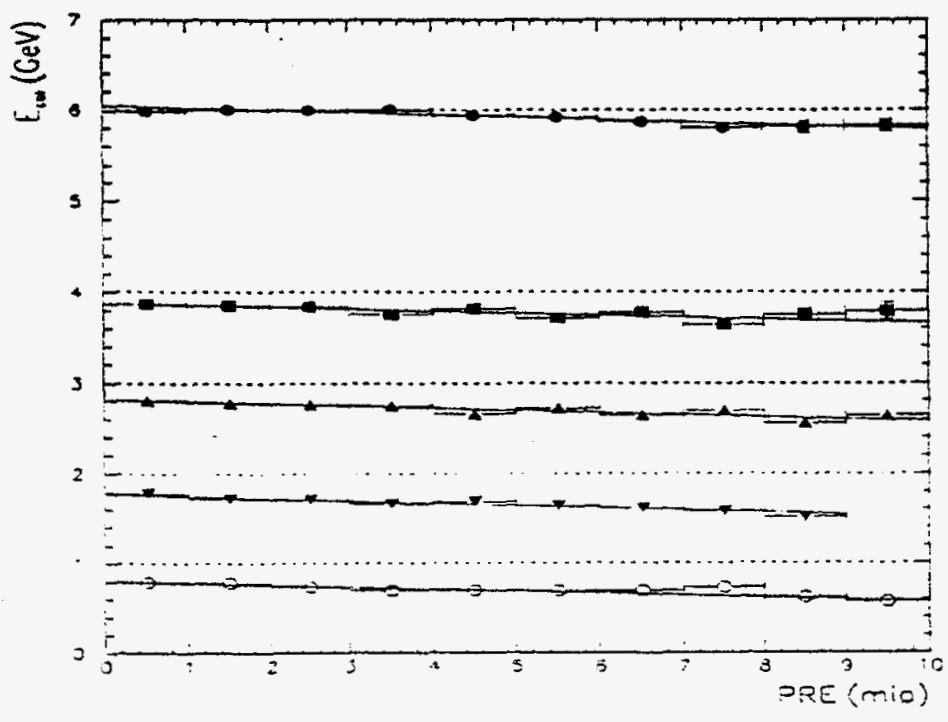

Figure 11: $\mathrm{BCAL}$ measured energy $\left(E_{c a l}\right)$ versus presampler signal (PRE) in mips for various beam energies. The solid lines are a straight line fit to the data and the dashed lines indicate the beam energies. 
The observed relationships can be parameterized by :

$$
E_{\text {cal }}=a_{0}+a_{1} E_{\text {pres }}
$$

where $E_{c a l}$ is the measured calorimeter energy and $E_{\text {pres }}$ is the measured presampler signal in mips. The parameters $a_{i}$ depend on the amount of material through which the particle passes and on its incident energy and so can be expressed as :

$$
a_{i}=\alpha_{i}+\beta_{i} E_{\text {beam }}
$$

where $\alpha_{i}$ and $\beta_{i}$ are parameters to be determined by fitting the calorimeter versus presampler curves for different energies at fixed material amounts and $E_{\text {beam }}$ is the beam energy of the particle incident on the inactive material. After determining the parameters $\alpha_{i}$ and $\beta_{i}$, the corrected calorimeter energy is given by :

$$
E_{\text {cal }}^{\text {cor }}=\frac{E_{\text {cal }}-\alpha_{0}-\alpha_{1} E_{\text {pres }}}{\beta_{0}+\beta_{1} E_{\text {pres }}}=E_{\text {beam }}
$$

The values of the parameters for the $1 X_{0}$ aluminum block are shown in Table 1 .

\begin{tabular}{|c|c|c|c|}
\hline$\alpha_{0}(\mathrm{GeV})$ & $\alpha_{1}(\mathrm{GeV} / \mathrm{mip})$ & $\beta_{0}$ & $\beta_{1}\left(\mathrm{mip}^{-1}\right)$ \\
\hline \hline-0.31 & -0.021 & 1.06 & -0.0009 \\
\hline
\end{tabular}

Table 1: Presampler energy correction parameters for $1 X_{0}$.

The values in Table 1 were used to correct the measured calorimeter energies for incident electrons. Similar results were also obtained for the correction factors to measured hadron (pions) energies. Figure 12 shows the electron $E / E_{\text {beam }}$ results from the calorimeter alone, together with the corrected values. In addition to correcting the measured calorimeter energies as shown in Figure 12, the correction procedure using $P R E$ aiso improves the measured energy resolutions slightly from an average of $22 \% / \sqrt{E}$ before correction to $20 \% / \sqrt{E}$ after correction.

\subsection{Electron/Pion Separation with the Presampler}

In the ZECS detector, between the interaction point and the BCAL modules, there is a superconducting coil, of nominal thickness $1 X_{0}$, that provides the magnetic field 


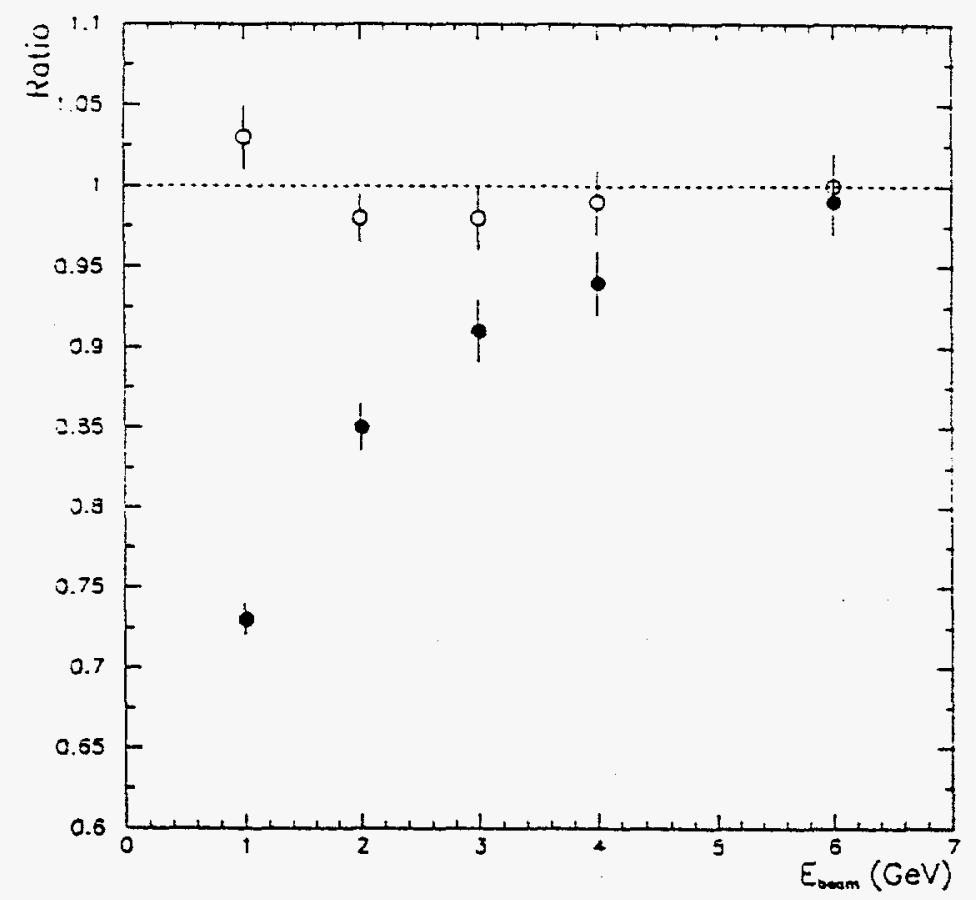

Figure 12: $E / E_{0}$ for measured calorimeter energies versus $E_{\text {beam }}$ with $1 X_{0}$ absorber (solid circles) and $E / E_{\text {beam }}$ after applying the presampler corrections (open circles).

for the detector. Because the low energy electrons are much more likely to begin showering in one radiation length than are the low energy hadrons, the presampler can be used to discriminate between the showered electrons and the nonshowered hadrons. As seen in Figure 10, the peak in the pion distribution is the signal from the unshowered pions and in this single mip region of the bistogram, the electron and pion distributions differ by about an order of magnitude.

Since the coil is $1 X_{0}$ thick, a high electron identification efficiency with good pion rejection is not possible as seen in Figure 13 which shows the dependence of the electron/pion separation on the presampler cut. For all beam momenta, the cut on the presampler pulse height was set by requiring a $75 \%$ electron identification. For example, for the $3 \mathrm{GeV} / \mathrm{c}$ electrons and pions shown in Figure 10, all events with a presampler signal greater than $203 \mathrm{ADC}$ counts were called electron events. This 


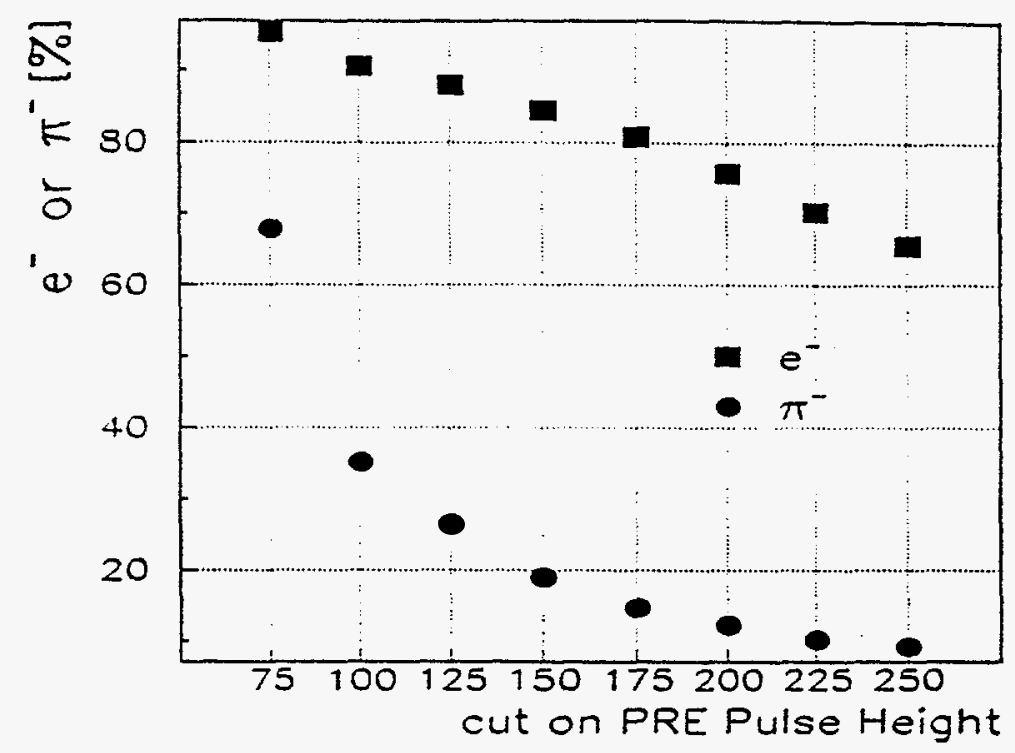

Figure 13: The electron identification and the pion acceptance as a function of the presampler pulse height cut where the cut identifies all events with presampler signal above the given value as electron events. The beam momentum is $3 \mathrm{GeV} / \mathrm{c}$.

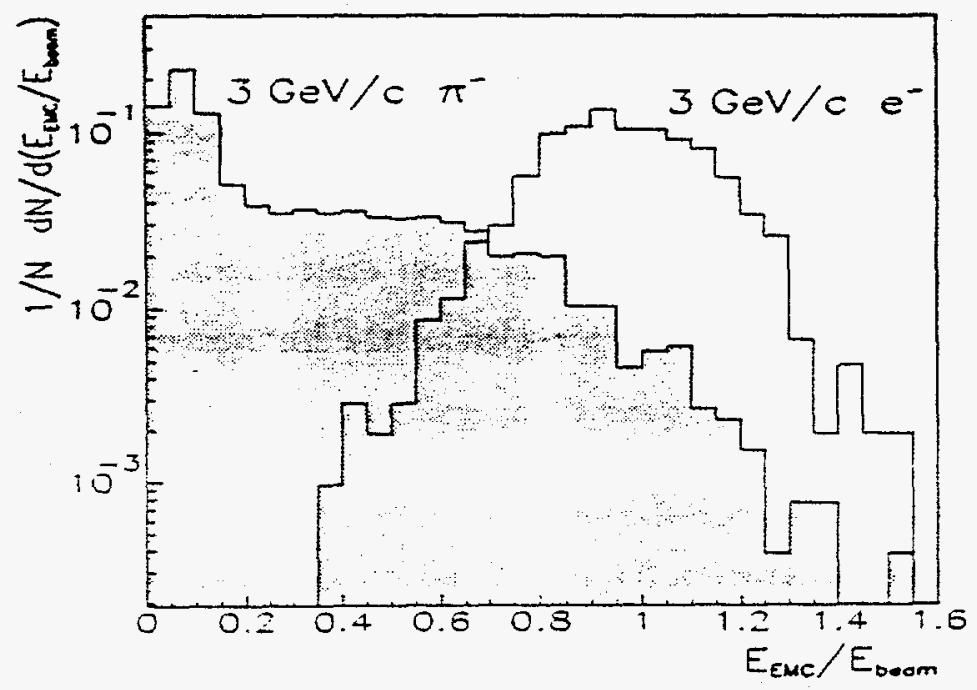

Figure 14: A histogram of the energy deposited in the EMC section of BCAL (E EMC) divided by the beam energy ( $E_{\text {beam }}$ ) for $3 \mathrm{GeV} / \mathrm{c}$ electrons (open histogram) and 3 $\mathrm{GeV} / \mathrm{c}$ pions (shaded histogram). 
resulted in $12.1 \%$ of the pions being accepted as electrons, as seen in Figure 13.

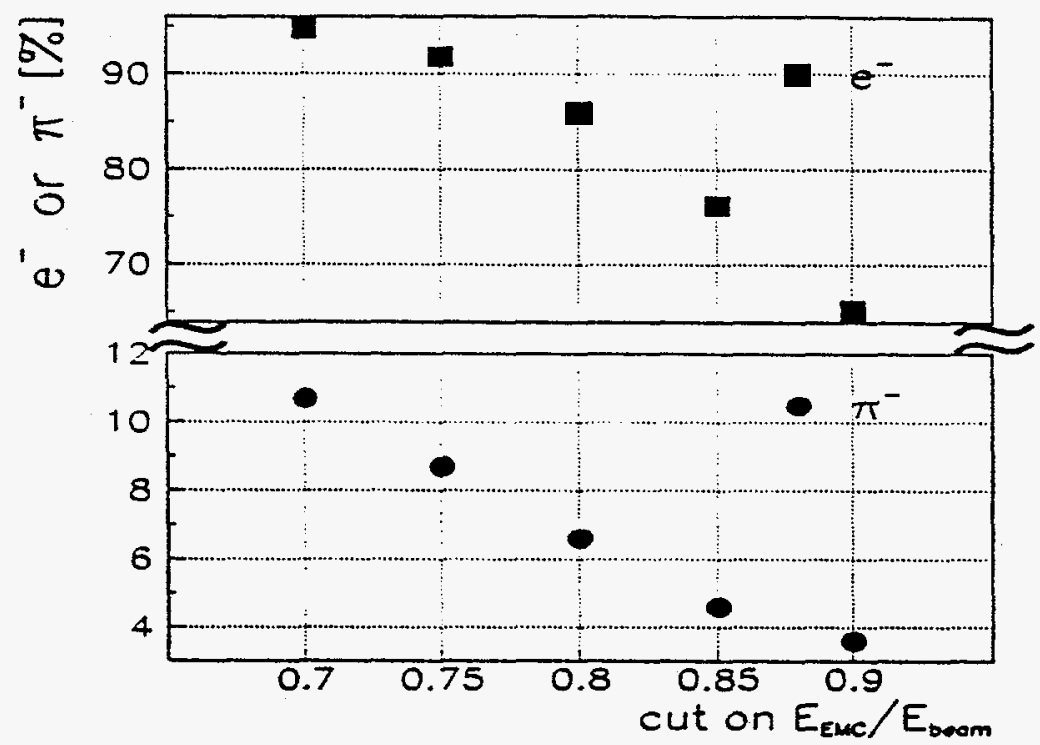

Figure 15: The electron identification and the pion acceptance as a function of the $B C A L$ cut on the fraction of energy deposited in the EMC section, $E_{E M C} / E_{\text {beam }}$. The beam momentum is $3 \mathrm{GeV} / \mathrm{c}$.

The BCAL itself can also be used to discriminate between electrons and pions. Histograms of the energy deposited in the EMC section of the BCAL divided by the beam energy, $\mathrm{E}_{E M C} / \mathrm{E}_{\text {beam }}$, are shown in Figure 14 for $3 \mathrm{GeV} / \mathrm{c}$ electrons and pions. Figure 15 shows the dependence of the electron/pion separation on the $\mathrm{E}_{E M C} / \mathrm{E}_{\text {beam }}$ calorimeter cut.

To allow a direct comparison between the presampler and the BCAL, a $75 \%$ electron identification is again chosen. For the histograms of Figure 14, this cut is at $E_{E M C} / E_{\text {beam }}=0.86$ and results in misidentification of $4.3 \%$ of the pions as electrons. Pion acceptances corresponding to $75 \%$ electron identification, for the other beam momenta using the PRE and the BCAL are shown in Figure 16.

The presampler and BCAL cuts can be variously combined to further improve the $\mathrm{e} / \pi$ ratio with results shown as the filled triangles in Figure 16 . The addition of 


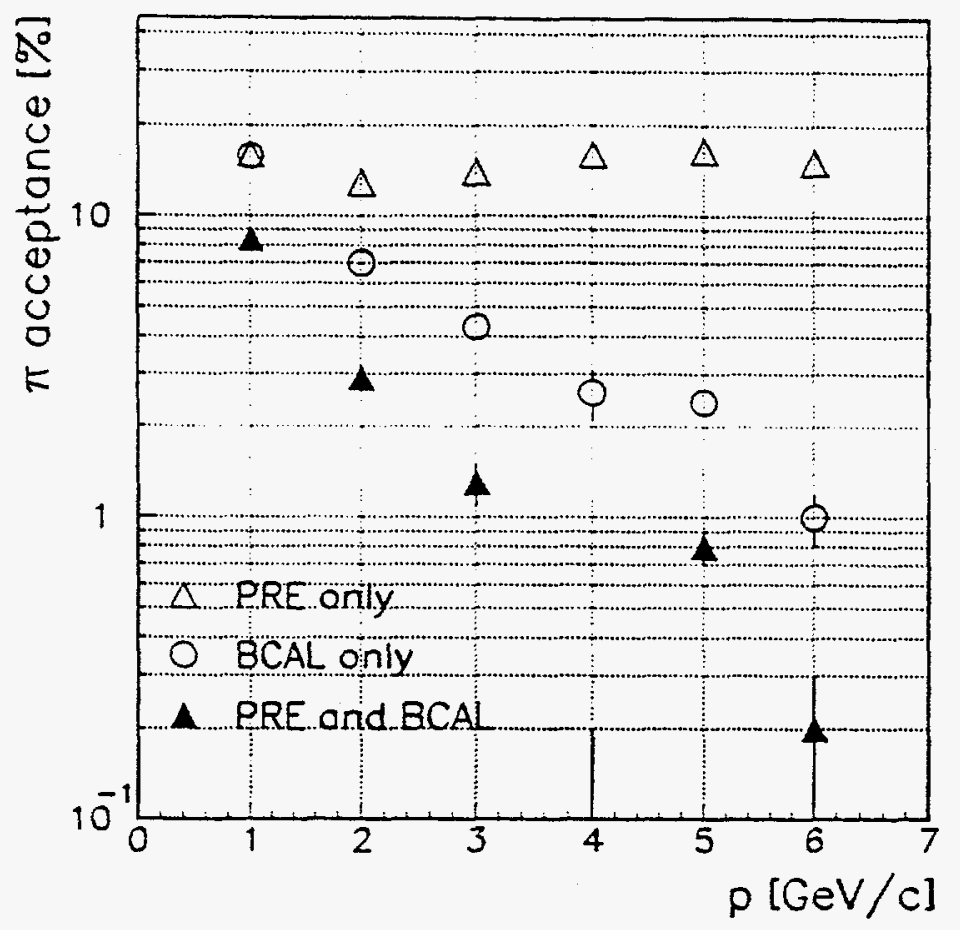

Figure 16: The pion acceptance for $75 \%$ electron identification for beam momenta from $1-6 \mathrm{GeV} / \mathrm{c}$ using the PRE and the BCAL.

the PRE improves the e/ $\pi$ rejection of the BCAL by a factor varying from two at 1 $\mathrm{GeV}$ to five at $6 \mathrm{GeV}$.

\section{BHES Results}

\subsection{Characteristics of the BHES Data}

The BHES is placed at a depth in the BCAL module where, on average, the number of particles in the electron shower is a maximum [11] and hadrons of the same energy are unlikely to have begun showering. The pedestal distribution for a single BHES wire has a tail coming from signals produced by the uranium radioactivity[12]. Figures $17(\mathrm{a})$ and $18(\mathrm{a})$ show the charge distribution of the pedestal on a typical 

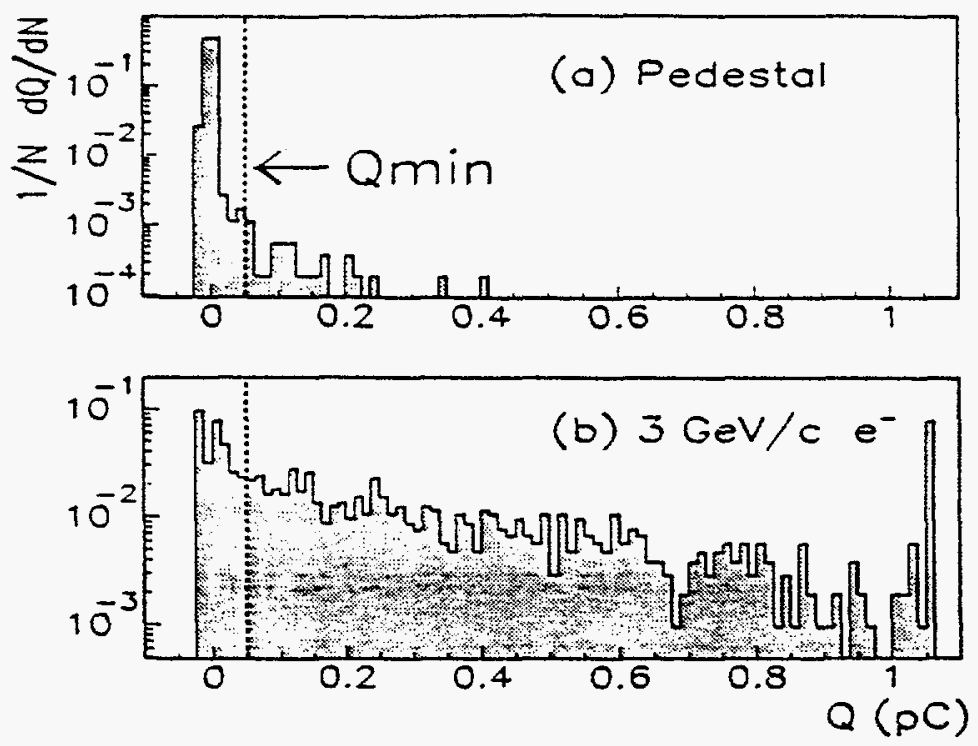

Figure 17: (a) Pedestal distribution for a typical BHES wire in units of charge with $A D C$ pedestal subtracted. (b) Signal from $3 \mathrm{GeV} / \mathrm{c}$ electrons on a single BHES wire. The $Q_{\min }$ cut at $0.05 \mathrm{pC}$ is indicated.
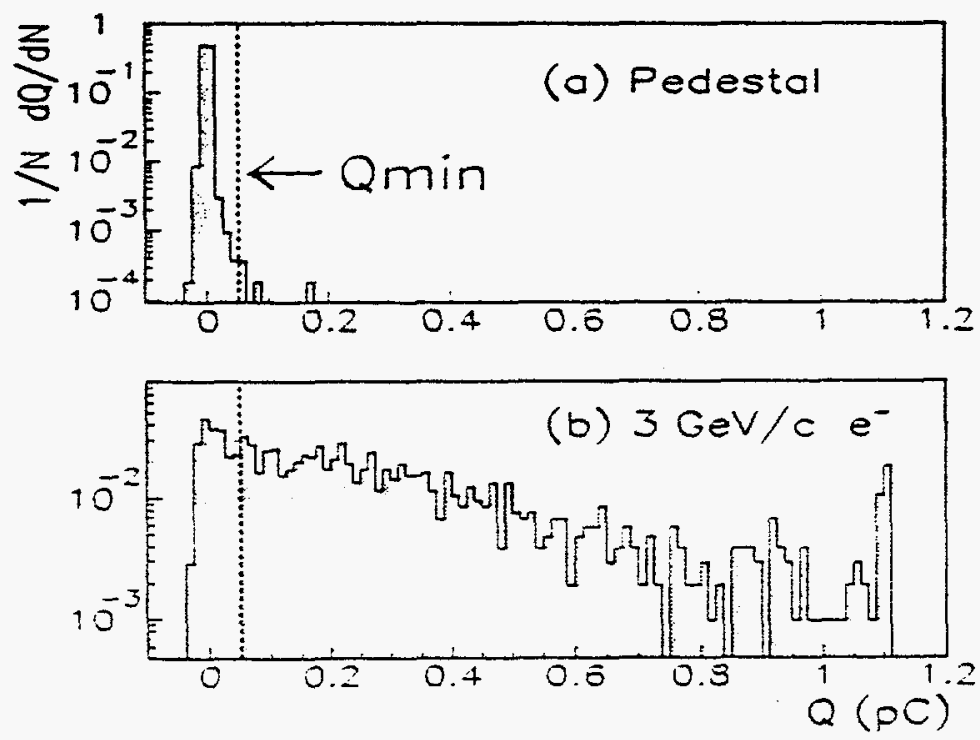

Figure 18: (a) Pedestal distribution for a typical BHES pad in units of charge with ADC pedestal subtracted. (b) Signal from $3 \mathrm{GeV} / \mathrm{c}$ electrons on a single $B$ HES pad. The $Q_{\min }$ cut at $0.05 \mathrm{pC}$ is indicated. 
BHES wire and pad respectively. Note the longer tail in the wire pedestal distribution which results from the larger effective area of the uranium plate seen by a wire as compared to a pad. From studies with muons we estimate the mean mip wire signal to be at $Q=0.07 \mathrm{pC}$.

Two quantities obtained from the BHES can be used to distinguish between showered electrons and non-showered pions. The first is the shower profile, measured by either the number of wires or the number of pads per event having signals above some minimum charge $Q_{\min }$. The second quantity is the sum of the charge, $Q_{\text {tot, }}$ on a contiguous group of either wires or pads that are centered on the incident particle. We choose five wires for the former analysis and three pads for the latter. A minimum charge requirement, $Q_{\min }$, is introduced to make the electron or pion signal less sensitive to the noise in the BHES coming from the uranium radioactivity. Signals from a $3 \mathrm{GeV} / \mathrm{c}$ electron beam, in units of charge, are shown in Figure 17(b) for a single BHES wire and in Figure 18(b) for a single pad. The value of $Q_{\min }$ used is indicated. The optimum value of $Q_{m i n}$ varies with beam momentum but is in the range 0.01 to $0.10 \mathrm{pC}$. The effect of the choice of $Q_{\min }$ on the two BHES quantities is discussed in Section 7.4.

As an example, a histogram of the number of wires per event with charge exceeding $0.05 \mathrm{pC}$, for a beam momentum of $3 \mathrm{GeV} / \mathrm{c}$, is shown in Figure 19(a) for electrons and Figure 19(b) for pions. There is a clear difference in the distributions. A histogram of the second quantity, $Q_{t o t}$, is shown in Figure 20 for both electrons and pions. Again there is a clear difference in shower activity as measured by the difference in the two distributions, with the pion signal peaking at low $Q$. These differences will be exploited in distinguishing electrons from pions in Section 7.1 .

\subsection{Pulse Height Variation Along a Wire}

The gain uniformity along a wire was studied at the test beam by recording wire pulse heights at eleven points over a distance of one meter. This beam scan was 

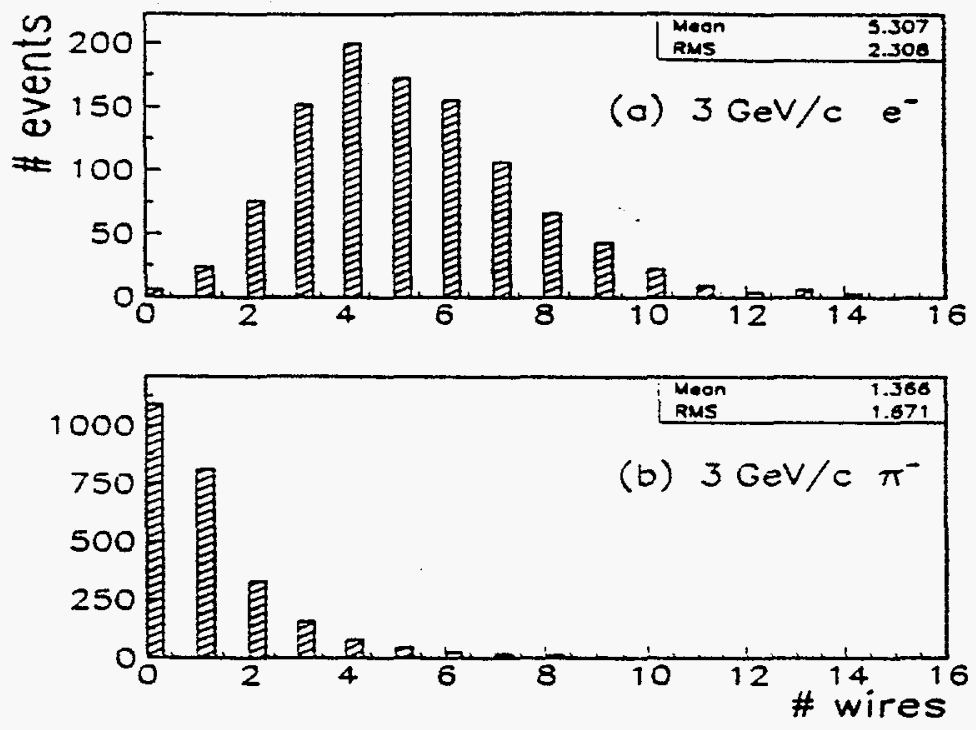

Figure 19: Number of wires per event with charge above $Q_{\min }=0.05 \mathrm{pC}$ for (a) 3 $\mathrm{GeV} / \mathrm{c}$ electrons and (b) $3 \mathrm{GeV} / \mathrm{c}$ pions.

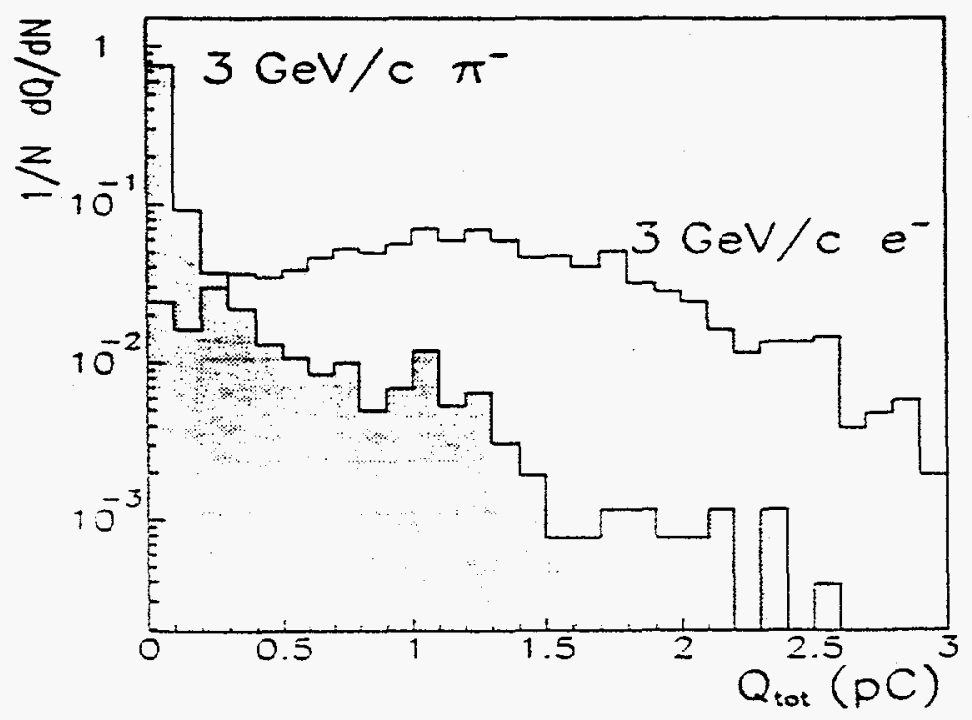

Figure 20: Total charge deposited on five wires about the beam, for $3 \mathrm{GeV} / c$ electrons (open histogram) and $3 \mathrm{GeV} / \mathrm{c}$ pions (shaded histogram). 
done at a momentum of $4 \mathrm{GeV} / \mathrm{c}$ with the electron trigger. Most of the shower energy in the proportional wire chamber is deposited in four wire cells. As seen from Figure 19, the sum over twelve wires represents a conservative shower profile and this ensured that the total shower energy was insensitive to the possibility that the detector did not move exactly in the horizontal plane. The shower energy, in $\mathrm{pC}$ of charge, as a function of position, is shown in Figure 21a. The maximum excursion of any measurement from the mean charge is less than $3.5 \%$. The lines denote the $\pm 10 \%$ limits from the mean charge. The uniformity along the four individual wires containing most of the shower charge is somewhat worse, as seen in Figure 21b - e, where the lines again denote the $\pm 10 \%$ limits from the mean charge.

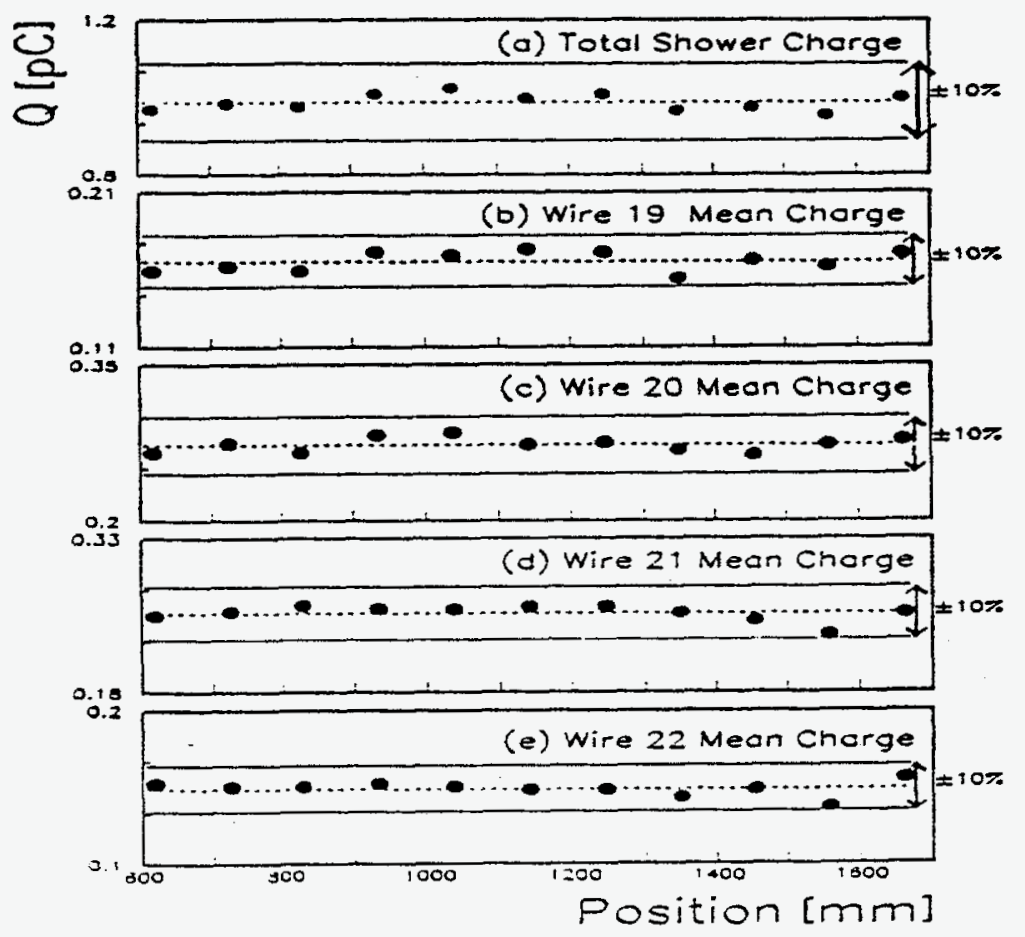

Figure 21: (a) The shower energy in $\mathrm{pC}$, deposited in twelve wire cells, as a function of position. (b) - (e)The shower energy in PC, deposited in four individual wire cells, as a function of position. The size of the data points indicate the errors bars. 


\subsection{Position Resolution}

With its finer sampling granularity in both the $z$ (pads) and $\phi$ (wires) coordinates, the BHES provides better position resolution in each coordinate as compared to the BCAL alone. Such an improvement is especially important for identifying the $\pi^{0} \rightarrow 2 \gamma$ decays and matching energy clusters with tracks reconstructed in the central tracking detector.

Using only the calorimeter information, the z-position of an electron in the calorimeter, $z_{\text {cal }}$, was calculated using the energy weighted EMC cell centroid,

$$
z_{\text {cal }}=\frac{\sum_{E M C_{\text {cells }}} E_{E M C_{\text {cell }}} \times Z_{E M C_{c e l l}}}{\sum_{E M C \text { cells }} E_{E M C_{c e l l}}}
$$

where $Z_{E M C \text { cell }}$ is the central position of each EMC cell and $E_{E M C \text { cell }}$ is the measured charge. The corresponding electron position measured by the BHES, both $y_{B B E S}$ and $z_{B H E S}$, using the wire and pad BHES signals were obtained by the same energy weighting method. To calculate these positions, only signals from the wires and pads near to the incident particle trajectory were used, since the contribution from the uranium noise far from the beam impact position can influence the measurement. The charges from a region of \pm 2 wires about the central wire of the beam profile, and a region of \pm 1 pad about the central pad, were summed.

The $y$ position (corresponding to $\phi$ in the detector), using only the calorimeter information, $y_{c a l}$, was calculated from the signals in the two PMT's that read out on each side of the module, sides $\mathrm{A}$ and $\mathrm{B}$. If $E_{A}, E_{B}$ are the corresponding calorimeter energy responses, then

$$
y_{c a l}=\text { Const. }+\frac{\lambda_{e f f}}{2} \ln \frac{E_{A}}{E_{B}}
$$

where $\lambda_{e f f}$ is $28 \mathrm{~cm}$. The beam was positioned $25 \mathrm{~mm}$ above ( $y$-coordinate) the center of the BCAL and centered on one EMC cell (z-coordinate). The $z_{\text {eal }}$ and $y_{c a l}$ positions were calculated for only those events where the shower was contained essentially in 
one EMC cell.
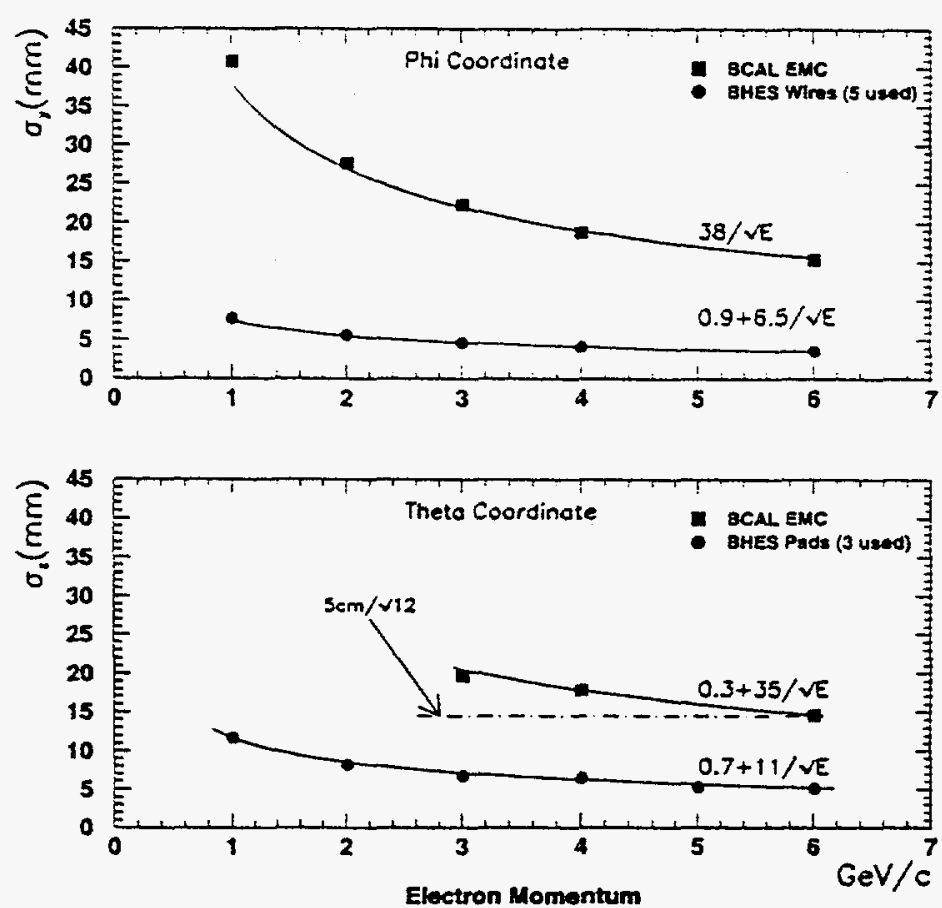

Figure 22: Position resolution in the $\phi$ and $\mathrm{z}$ directions for both the calorimeter and the BHES. The single cell calorimeter resolution of $5 / \mathrm{sqrt}(12) \mathrm{cm}$ is indicated. The width of the beam was not subtracted.

Figure 22 compares the resolution of position measurements made with the calorimeter with those from the BHES in the $\phi$ direction ( $y$-coordinate) and the $z$ direction, as a function of energy. The resolution is defined as the RMS of the distributions in $y_{c a l}\left(z_{c a l}\right)$ or $y_{B H E S}\left(z_{B H E S}\right)$ as defined above. At low energies the improvement in $\phi$ is about a factor of five, falling to about a factor of three at 6 $\mathrm{GeV} / \mathrm{c}$. In the $\mathrm{z}$ direction, the improvement is not as dramatic, since the relative size of the pads and the EMC towers is only three. 


\subsection{Electron/Pion Separation with the BHES.}

The analysis focuses on using either pads alone or wires alone as elements in determining the structure of the events. In the following examples, only distributions from the wires are shown for brevity.

Two cuts are introduced to distinguish between electrons and pions using the quantities discussed in Section 7.1. The first cut is on the number of elements (wires

or pads) per event with charge above $Q_{\min }$. For wires, the dependence of the pion rejection on the placement of this first cut alone for a beam momentum of $3 \mathrm{GeV} / \mathrm{c}$ and $Q_{\min }=0.05 \mathrm{pC}$ is shown in Figure 23. In analyzing the data, for all beam momenta, this cut is placed such that all events with more than one wire having a signal above $Q_{\min }$ are called electron events. For example, at $3 \mathrm{GeV} / \mathrm{c}$ this cut correctly identifies $97.1 \%$ of the electrons as electrons and misidentifies $26.9 \%$ of the pions as electrons. For completeness, Figure 24 shows the sensitivity of this cut to the choice of $Q_{\text {min }}$.

The second cut is on the element charge collected per event, $Q_{\text {tot }}$. The dependence of the $e / \pi$ separation on the placement of this second cut alone, for a beam momentum of $3 \mathrm{GeV} / \mathrm{c}$, is shown in Figure 25. The placement of the cut on $Q_{\text {tot }}$ varies with the beam momentum and at $3 \mathrm{GeV} / \mathrm{c}$ it is placed such that all events that deposit more than $0.35 \mathrm{pC}$ of charge on the wires are called electron events. This correctly identifies $91.5 \%$ of the electrons and misidentifies $10.0 \%$ of the pions as electrons. Figure 26 shows the cut on $Q_{\text {tot }}$ is relatively insensitive to the choice of $Q_{\text {min }}$. The above two cuts can be combined to produce an electron identification of $90 \%$ and for particles of momentum $3 \mathrm{GeV} / \mathrm{c}$, this results in a pion misidentification as an electron of $8.4 \%$.

The values of $Q_{\min }$ and $Q_{\text {tot }}$ need to be tuned separately for either pad elements or wire elements, as expected, since the pads collect four times less charge than the wires. By varying these two quantities we can determine the pion rejection at given electron identification efficiencies. Figure 27 shows the variation of pion acceptance with electron identification using either pad elements or wire elements in the analysis. 


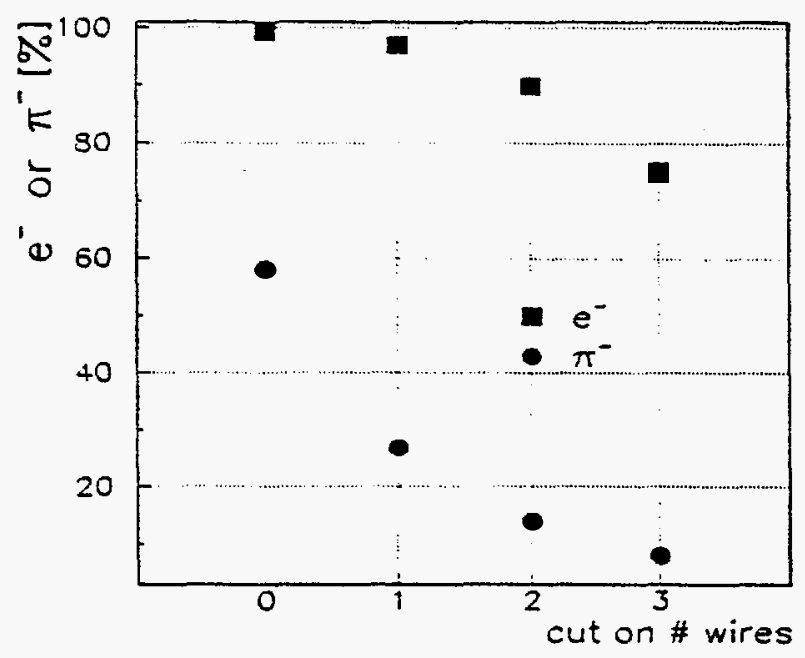

Figure 23: The electron identification and pion acceptance as a function of the BHES cut on the number of wires per event with charge above $Q_{\min }=0.05 \mathrm{pC}$. The cut requires that for an event to be identified as an electron event the number of wires must be greater than the given value. The beam momentum is $3 \mathrm{GeV} / \mathrm{c}$.

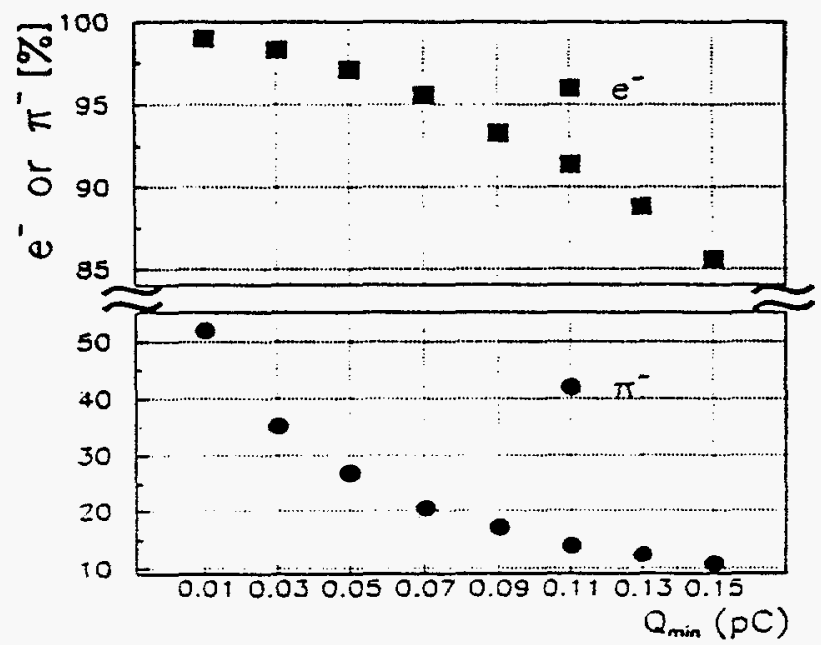

Figure 24: The electron identification and pion acceptance as a function of $Q_{\min }$ for a fixed BEES cut on the number of wires per event (All events with more than one wire with signal above $Q_{\min }$ are called electron events). The beam momentum is 3 $\mathrm{GeV} / \mathrm{c}$. 


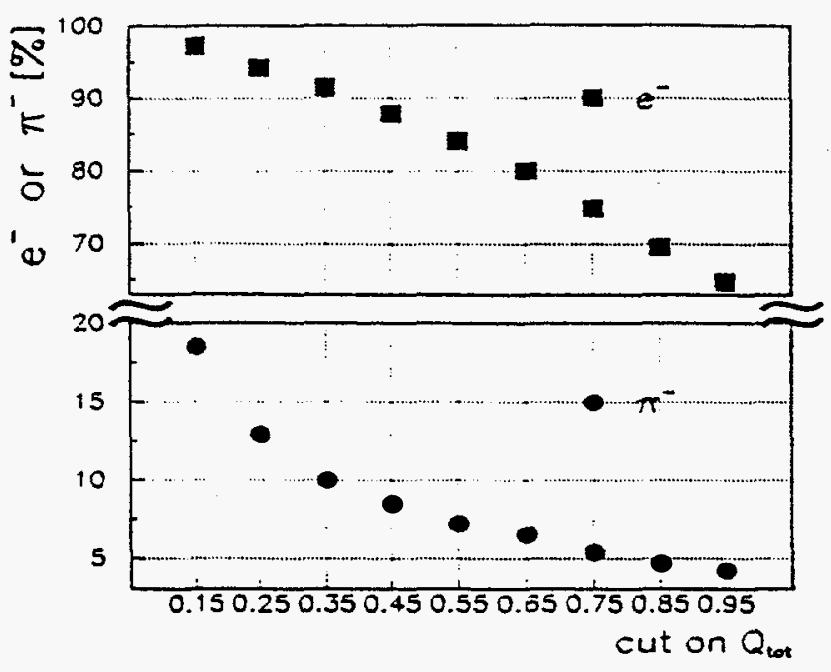

Figure 25: The electron identification and pion acceptance as a function of the BHES cut on $Q_{\text {tot. }} Q_{\text {tot }}$ is the charge deposited on the five wires centered on the incident particle and the cut requires that for an event to be identified as an electron event $Q_{\text {tot }}$ must be greater than the given value. The beam momentum is $3 \mathrm{GeV} / \mathrm{c}$.

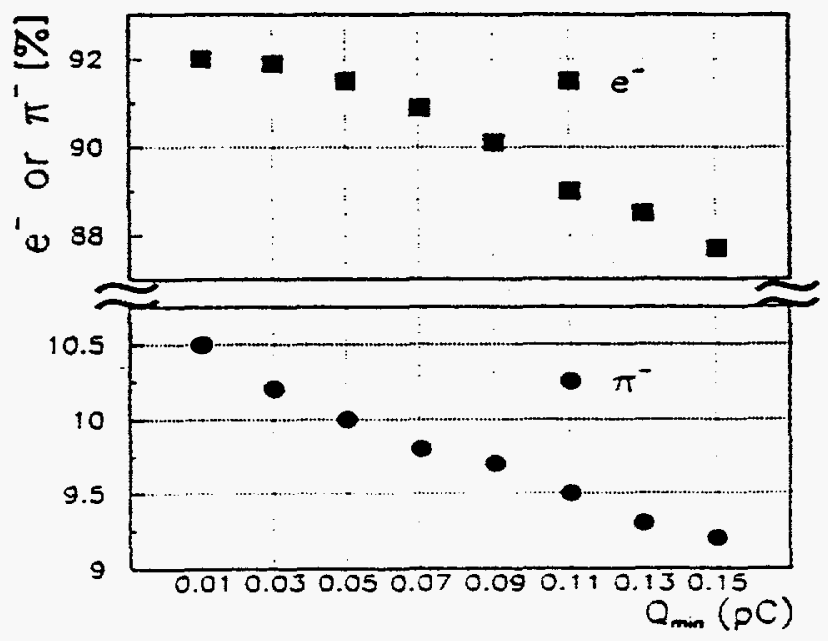

Figure 26: The electron identification and pion acceptance as a function of $Q_{\min }$ for a fixed BHES cut on $Q_{\text {tot }}$. (All events with $Q_{\text {tot }}$ greater than $0.35 \mathrm{pC}$ are called electron events.) The beam momentum is $3 \mathrm{GeV} / \mathrm{c}$. 


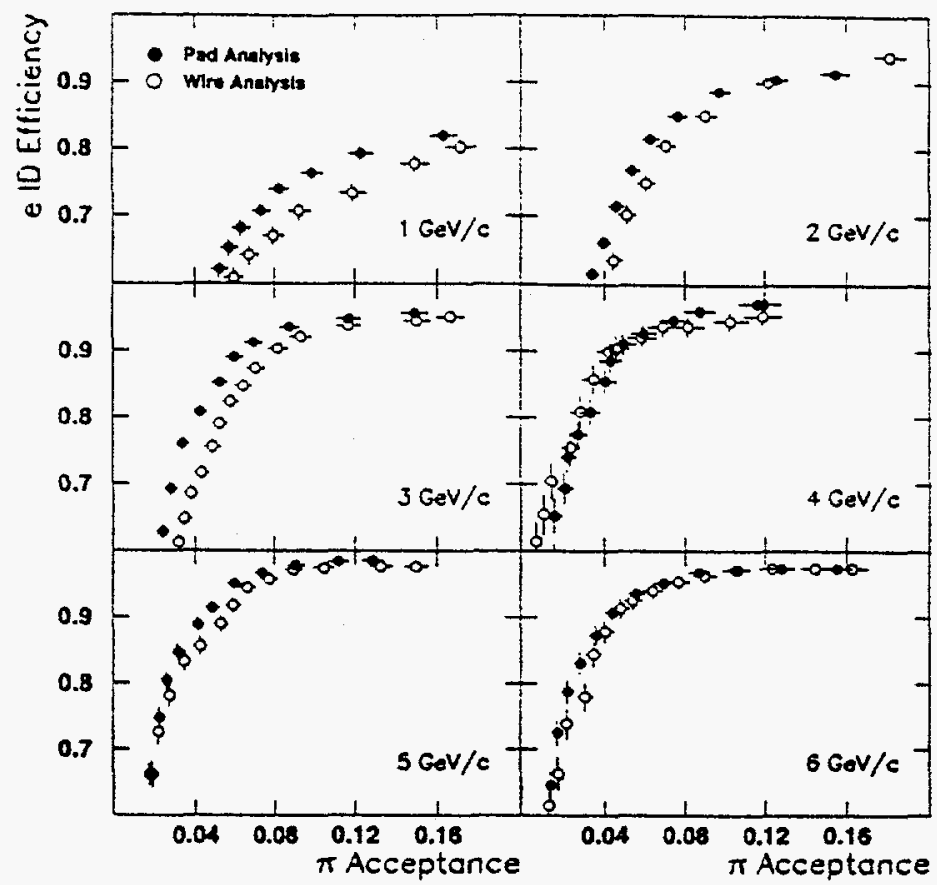

Figure 27: Pion acceptance as a function of electron identification efficiency using either pads (closed circles) or wires (open circles) to determine the structure of the events.

Using pads to determine the event structure results in better pion rejection at low momenta. The pion misidentification depends on how much of the pion signal extends into the higher charge region. Since a wire sees about five times more DU noise than a pad, the noise contribution to the pion signal for wires is larger than that for pads. Thus we expect the pads to give better separation than the wires, as is observed.

Pion acceptances for beam momenta between 1 and $6 \mathrm{GeV} / \mathrm{c}$ are shown in Figure 28 for $90 \%$ electron identification and in Figure 29 for $75 \%$ electron identifcation. In Figure 28, to achieve $90 \%$ electron identification at $1 \mathrm{GeV} / \mathrm{c}$, the analysis was modified so that all wires with charge above $Q_{\min }$ were included in the sum to calculate $Q_{\text {tot }}$ and all events with one or more wires with charge greater than $Q_{\min }$ 
were called electron events.

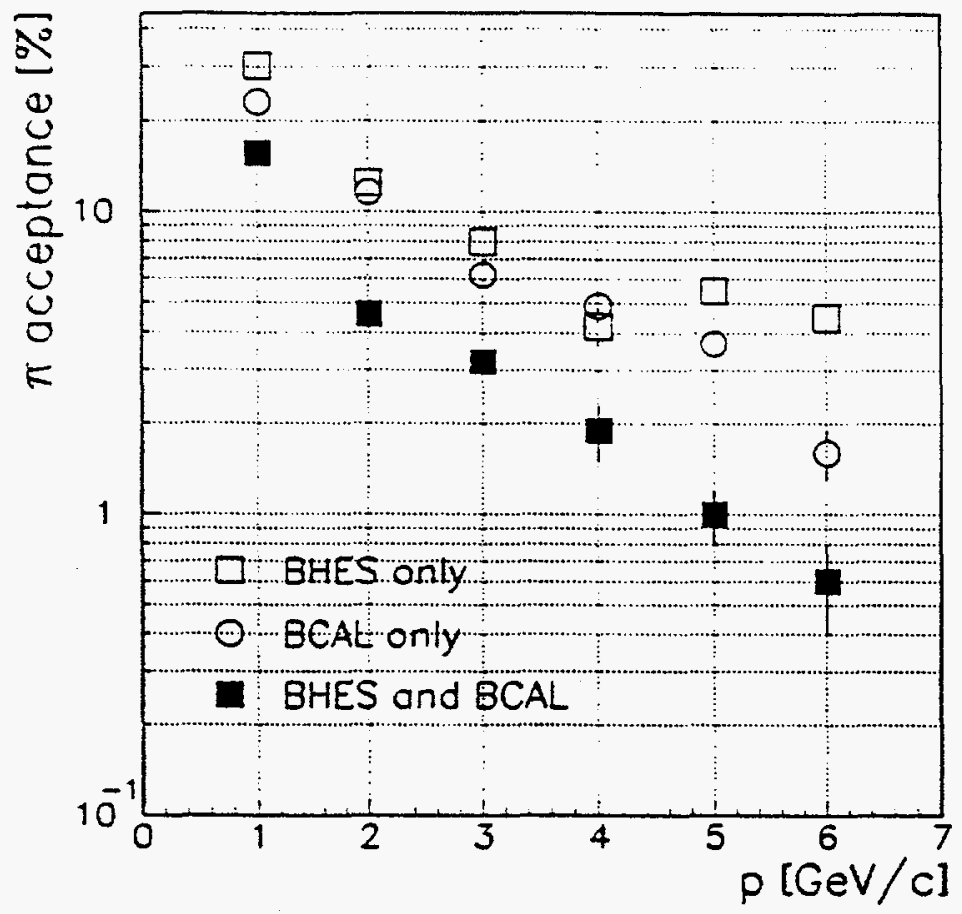

Figure 28: The pion acceptance for $90 \%$ electron identification for beam momenta from $1-6 \mathrm{GeV} / \mathrm{c}$ using the BHES and the BCAL.

The BCAL can again be used to discriminate between electrons and pions in the same way as described in Section 6.2. The pion acceptance, using the BCAL only, as a function of the beam momentum is shown in Figure 28 for $90 \%$ electron identification and in Figure 29 for $75 \%$ electron identification. For a beam momentum of $3 \mathrm{GeV} / \mathrm{c}$, the pion misidentification is $6.2 \%$ and $4.3 \%$ respectively.

We can now use the BHES and the BCAL together to improve the $e / \pi$ discrimination by requiring that electron candidates satisfy the requirements imposed by both detectors. For a beam momentum of $3 \mathrm{GeV} / \mathrm{c}$ and an electron identification of $90 \%$, combining the BHES and the BCAL cuts reduces the pion acceptance as an electron from $6.2 \%$ to $3.2 \%$. Similarly, for $75 \%$ electron identification the reduction 
is from $3.2 \%$ to $0.9 \%$ when the BHES is included. The pion acceptances, using both the BCAL and the BHES, as a function of the beam momentum for $90 \%$ and $75 \%$ electron identification are also shown in Figure 28 and Figure 29.

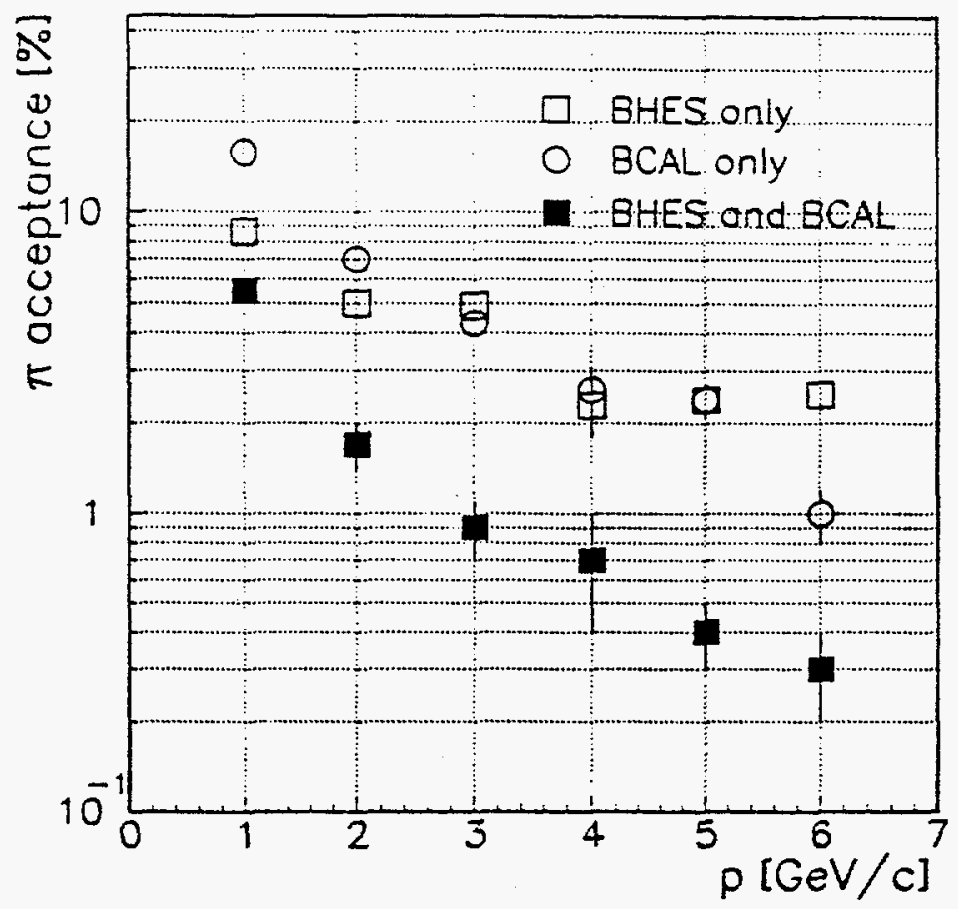

Figure 29: The pion acceptance for $75 \%$ electron identification for beam momenta from $1-6 \mathrm{GeV} / \mathrm{c}$ using the BHES and the BCAL. To obtain the $1 \mathrm{GeV} / \mathrm{c}$ and 2 $\mathrm{GeV} / \mathrm{c}, \mathrm{BHES}$-only and BHES-and-BCAL data points, the BHES pads were used and to obtain the data points at the remaining momenta the BHES wires were used.

The $e / \pi$ discrimination shown in these figures was determined by using pads or wires to discriminate the event structure. Although pads by themselves yield better rejection than wires by themselves, there is no advantage to using pads over wires in conjunction with the BCAL at energies above $2 \mathrm{GeV}$.

The combination of BHES and BCAL is about a factor of two more efficient at rejecting pions than the PRE and BCAL as can be seen by comparing Figures 29 and 16. 


\section{Conclusions}

The addition of a presampler and shower max detector will significantly improve the performance of the ZEUS Barrel Calorimeter. The presampler allows energy corrections for electrons and jets to be made on an event by event basis and so reduces the systematic errors coming from jet fragmentation fluctuations. It also significantly improves the pion rejection at $75 \%$ electron acceptance over the use of the BCAL alone.

The BHES gives a major improvement in the position measurement of electromagnetic showers, so allowing the match to potential electron tracks to be made with higher precision in both transverse directions. Monte Carlo studies show that this discrimination, when combined with the improved $e / \pi$ separation resulting from the HES signals, improves the electron identification in heavy quark jets by about a factor of five.

\section{Acknowledgments}

We would like to thank the Ohio State ZEUS group, D. Acosta, L.S. Durkin, J. Gilmore, and W.N. Murray for their work in the testbeam setup, John Dawson of Argonne National Laboratory for the design of the BHES readout system and R. Loveless of the University of Wisconsin for help in setting up the support stand. We also thank Brookhaven National Laboratory for the use of their testbeam, and in particular D. Dayton and A. Carroll. This work was supported by the U.S. Department of Energy, Division of High Energy Physics, under contract No. W-31-109-ENG-38.

\section{References}

[1] U. Behrens et al, NIM A289, (1990) 115.

[2] A. Andresen et al, NIM A309, (1991) 101. 
[3] M. Derrick et al, NIM A309, (1991) 77.

[4] A. Dwurazny et al, NIM A277, (1989) 176.

[5] L. Nodulman et al, NIM 176, (1980) 345.

[6] D. Acosta et al, NIM A354, (1995) 296.

[7] ZEUS Collaboration, DESY PRC 94-01, Jan(1994).

[8] A. Bernstein et al, NIM A336, (1993) 23.

[9] I. Ambats et al, NIM A320, (1992) 161.

[10] F. Sauli, CERN Report 77-09 (1977).

[11] D. Muller, Phys. Rev. $\underline{\mathbf{5}}_{\mathbf{2}}(1972) 2677$.

[12] PWC detectors have been previously used in a depleted uranium calorimeter at LEP and the effect of the uranium noise on a single mip signal was studied. Yu. Galaktionov et al, NIM A251, (1986) 258.

A. Arefiev et al, NIM A245, (1986) 71.

\section{DISCLAIMER}

This report was prepared as an account of work sponsored by an agency of the United States Government. Neither the United States Government nor any agency thereof, nor any of their employees, makes any warranty, express or implied, or assumes any legal liability or responsibility for the accuracy, completeness, or usefulness of any information, apparatus, product, or process disclosed, or represents that its use would not infringe privately owned rights. Reference herein to any specific commercial product, process, or service by trade name, trademark, manufacturer, or otherwise does not necessarily constitute or imply its endorsement, recommendation, or favoring by the United States Government or any agency thereof. The views and opinions of authors expressed herein do not necessarily state or reflect those of the United States Government or any agency thereof. 